\title{
Asymptotically Stable Walking for Biped Robots: Analysis via Systems with Impulse Effects
}

\author{
Jesse W. Grizzle, Gabriel Abba, and Franck Plestan
}

\begin{abstract}
Biped robots form a subclass of legged or walking robots. The study of mechanical legged motion has been motivated by its potential use as a means of locomotion in rough terrain, as well as its potential benefits to prothesis development and testing. This paper concentrates on issues related to the automatic control of biped robots. More precisely, its primary goal is to contribute a means to prove asymptotically-stable walking in planar, under actuated biped robot models. Since normal walking can be viewed as a periodic solution of the robot model, the method of Poincaré sections is the natural means to study asymptotic stability of a walking cycle. However, due to the complexity of the associated dynamic models, this approach has had limited success. The principal contribution of the present work is to show that the control strategy can be designed in a way that greatly simplifies the application of the method of Poincaré to a class of biped models, and, in fact, to reduce the stability assessment problem to the calculation of a continuous map from a subinterval of $\mathbb{R}$ to itself. The mapping in question is directly computable from a simulation model. The stability analysis is based on a careful formulation of the robot model as a system with impulse effects and the extension of the method of Poincaré sections to this class of models.
\end{abstract}

Index Terms-Control systems, limit cycles, mechanical systems, nonlinear systems, robot dynamics.

\section{INTRODUCTION}

$\mathbf{M}$ ECHANICAL biped locomotion has been studied for well over 30 years. A broad overview of the state of the art, until 1990, can be found in [46], [58], and [19], along with motivation for studying this class of electro-mechanical systems. The available literature addresses a wide range of topics, from model formulation, efficient means of computing the dynamical equations, relations between mechanical legged locomotion and biological legged locomotion, methods of synthesizing gaits, the mechanical realization of biped robots, and control.

One can distinguish several control design approaches from the literature. By far, the most common approach to control is through the tracking of precomputed reference trajectories. The trajectories may be determined via analogy, either with

Manuscript received February 24, 1999; revised October 1, 1999. The work of J. W. Grizzle was supported in part by the National Science Foundation under Grant ECS-9631237 and in part by a gift from the Ford Motor Company.

J. W. Grizzle is with the Control Systems Laboratory, Electrical Engineering and Computer Science Department, University of Michigan, Ann Arbor, MI 48109-2122 USA (e-mail: grizzle@umich.edu).

G. Abba is with LGIPM, Universit de Metz, IUT de Thionville, Espace Cormontaigne, 57970 Yutz, France (e-mail: abba@iut.univ-metz.fr).

F. Plestan is with IRCCyN, Ecole Centrale de Nantes, 44321 Nantes cedex 03, France (e-mail: Franck.Plestan@irccyn.ec-nantes.fr).

Publisher Item Identifier S 0018-9286(01)00295-1. biological systems [58], [1], or with simpler, passive, ${ }^{1}$ mechanical-biped systems [38], [56], [57]. They can be generated by an oscillator, such as van der Pol's oscillator [33], or computed through optimization of various cost criteria, such as minimum expended control energy over a walking cycle [10], [11], [14], [48],[49]. Within the context of tracking, many different control methods have been explored, including continuous-time methods based on PID controllers, [18], [19], [42] computed torque and sliding mode control [42], [13], [39], [47], [36], or essentially discrete-time methods, based on impulse control [14]. Other control methods have been investigated that do not rely on precomputed reference trajectories for the angular positions. These include controlling energy, angular momentum, and others [46], [50], [35], [26], [27], [20], [36], [45], [16]. The control design proposed here will not rely on precomputed reference trajectories.

To date, for the case of an under actuated biped robot with a torso, none of the various control approaches have produced a closed-loop system with provable stability properties. Proving stability is the primary goal of this paper. Since regular walking can be viewed as a periodic solution of the robot model, the method of Poincare sections is the natural means to study asymptotic stability of a walking cycle. However, due to the complexity of the associated dynamic models, this approach has only been applied successfully to Raibert's one-legged hopper [35], [12], [16], a biped robot without a torso [56], [20], [53], and to a fully actuated biped (walking) model in [26], [27]. One of the principal contributions of the present work is to show that the control strategy can be designed in a way that greatly simplifies the application of the method of Poincare to a class of under actuated biped models.

The stability analysis is built up in several steps. Section II presents the dynamic model of an underactuated biped robot with a torso, walking on a level surface. The model includes two important parts: a mechanical model that is valid when one leg is touching the ground (supporting the robot) and the other is free (i.e., not touching the walking surface), and an impulse model of the contact event (the swing leg touching the ground). The model used here is representative of many biped models found in the literature [56], [15], [25], [57], [29], [21]. The main contribution of this section is the formulation of the biped model as a nonlinear system with impulse effects [3], [59], which will be the basis for all of the analysis that follows.

The main contribution of Section III is the extension of the method of Poincaré sections to systems with impulse effects. The extension will be done in sufficient generality that it is ap-

\footnotetext{
${ }^{1}$ Here, passive is used in the sense that the system is not actuated, but can walk down an inclined plane.
} 
plicable to more complex robots than the one treated in Section II. Roughly speaking, the method of Poincaré sections entails finding a (local) hyper-plane that is transversal to a candidate periodic motion of a continuous-time system, and then inducing a discrete-time mapping form the plane to itself [43], [34]. The mapping, called the Poincaré return map, is defined by following the evolution of a trajectory of the continuous-time system from a point on the plane to its next intersection with the plane. Periodic motions of the continuous-time system correspond to fixed points of the induced map. In the case of a biped robot, there is a natural plane to use in the analysis, namely, the constraints corresponding to an impact with the walking surface. The principal result of Section IV is to show that the freedom in the control design can be used to reduce the stability assessment problem via the method of Poincaré to the (numerical) calculation of a continuous map from a subinterval of $\mathbb{R}$ to itself. This will be achieved with the use of finite-time stabilizing feedback controllers [23], [4]-[6]. The mapping in question, which is a restriction of the Poincaré return map, is directly computable from a simulation model of the closed-loop system. This results in a sufficiently tractable characterization of asymptotic stability that it can be efficiently incorporated into a (numerical) feedback design scheme for computing an asymptotically stabilizing feedback controller with low peak torque demands and good efficiency with respect to average energy consumption over a cycle [22], or to optimize the mechanical parameters of the robot itself.

Section V analyzes the internal behavior of the robot model in closed loop with a finite-time stabilizing controller, as the gain of the controller tends to infinity. Under bounded control gains, the classical zero dynamics of the mechanical part of the robot model are not invariant under the impact model, and hence cannot be used to analyze any of the asymptotic properties of the closed-loop system. However, in the high gain limit, the invariance of the zero dynamics is recovered. This can be used to explain certain properties of the Poincaré map.

It is emphasized that all of the above will be illustrated on one of the simplest biped robot models possible. The robot consists of a torso, hips, and two legs of equal length, with no ankles and no knees. The two legs are actuated. The reason for this choice of model is two fold. First, asymptotically stable walking has never been proved for such a model, and thus this simplest problem is still open [15]. Second, from a pragmatic standpoint, it did not seem advantageous to obscure the main elements of the control approach with the computational complexity of a more complete biped model.

\section{A SiMPLE BIPED MODEL}

This section introduces the dynamic model of a simple, planar biped robot. The robot consists of a torso, hips, and two legs of equal length, with no ankles and no knees. Thus, it has five degrees of freedom. Two torques are applied between the legs and the torso, so the system is under actuated. It is assumed that the walking cycle takes place in the sagittal plane and on a level surface. It is further assumed that the walking cycle consists of successive phases of single support (meaning only one leg is touching the ground), with the transition from one leg to an- other taking place in an infinitesimal length of time [49], [52], [15]. This assumption entails the use of a rigid model to describe the impact of the swing leg with the ground. The model of the biped robot thus consists of two parts: the differential equations describing the dynamics of the robot during the swing phase, and an impulse model of the contact event. Such models are very common in the field of biped locomotion. The only contribution made here will be the formulation of the model as a nonlinear system with impulse effects [3], [59], which will set up the model for the analysis to follow.

During the swing phase, the stance leg is modeled as a pivot. ${ }^{2}$ In order to avoid the swing leg scuffing the ground until the desired moment of contact, the idea of [38] is adopted here: the swing leg is assumed to move out of the plane of forward motion, and into the frontal (coronal) plane. This allows the swing leg to clear the ground and be posed in front of the stance leg (think of a person with a cast over their knee). It will be further assumed that the swing leg is designed to renter the plane of motion when the angle of the stance leg attains a given value, $\theta_{1}^{d}$. Alternate means of achieving leg clearance in stiff legged robots are discussed in [38], [15].

\section{A. Mechanical (Swing Phase) Model}

During the swing phase of the motion, the stance leg is acting as a pivot, and thus there are only three degrees of freedom. The definition of the angular coordinates and the disposition of the masses of the legs, hips and torso are indicated in Fig. 1. In particular, note that all masses are lumped, and positive angles are computed clockwise with respect to the indicated vertical lines. Two torques, $u_{1}$ and $u_{2}$, are applied between the torso and the stance leg, and the torso and the swing leg, respectively. The dynamic model of the robot between successive impacts is easily derived using the method of Lagrange [54]. This results in a standard second order system

$$
D(\theta) \ddot{\theta}+C(\theta) \dot{\theta}+G(\theta)=B(\theta) u
$$

where $u=\left(u_{1}, u_{2}\right)^{\prime}$, and $\theta=\left(\theta_{1}, \theta_{2}, \theta_{3}\right)^{\prime}: \theta_{1}$ parameterizes the stance leg, $\theta_{2}$ the swing leg and $\theta_{3}$ the torso. The matrices $D, C, G$, and $B$ are given in Appendix A.

The second-order system (1) can be written in state-space form by defining

$$
\begin{aligned}
\dot{x} & :=\frac{d}{d t}\left[\begin{array}{c}
\theta \\
\omega
\end{array}\right]=\left[\begin{array}{c}
\omega \\
D^{-1}(\theta)(-C(\theta, \omega) \omega-G(\theta)+B u)
\end{array}\right] \\
& =: f(x)+g(x) u .
\end{aligned}
$$

The state space for the system is taken as $\mathcal{X}:=\{x:=$ $\left.\left(\theta^{\prime}, \omega^{\prime}\right)^{\prime} \mid \theta \in M, \omega \in \mathbb{R}^{3}\right\}$, where $M=(-\pi, \pi)^{3}$. Of course, not all points in $M$ correspond to physically reasonable configurations of the robot (e.g., the robot being above the walking surface). One possibility, therefore, is to further restrict the admissible solutions through viability constraints [2], [8]. This would be an important additional consideration for kneed-bipeds, but for the simple stiff-legged model analyzed here, it is enough to initialize the model in a physically rea-

\footnotetext{
${ }^{2}$ Reference [17] shows how to compute the forces acting on the stance leg, and how to verify that the leg does not slip.
} 


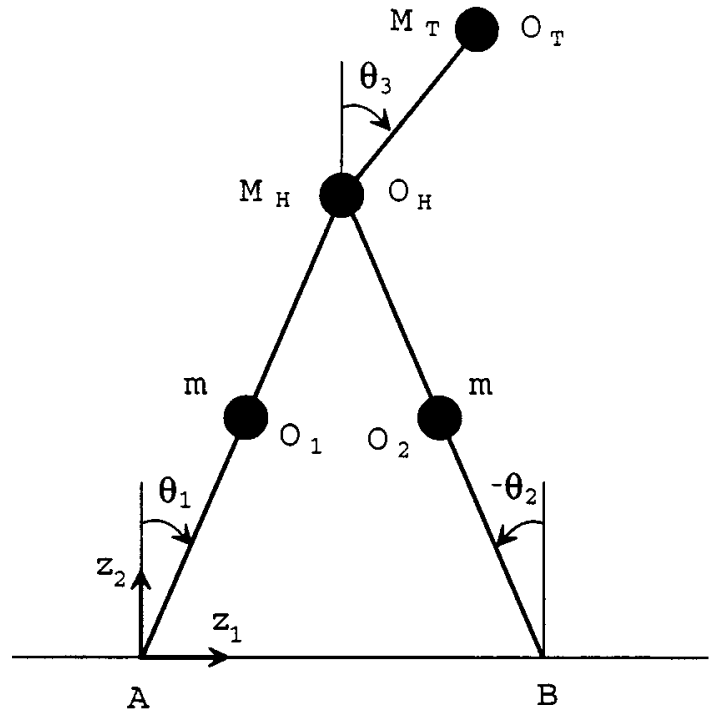

Fig. 1. Schematic indicating the defintion of the generalized coordinates and the mechanical data of the biped robot. All masses are lumped. The legs are symmetric, with length $r$ equal to the length of the line segment $A-O_{H}$ (also, $\left.B-O_{H}\right)$. The mass of each leg is lumped at $r / 2$. The distance from the center of gravity of the hips to the center of gravity of the torso, denoted by $l$, is the distance from $O_{H}$ to $O_{T}$.

sonable configuration and allow the impact model (see later) to maintain the trajectory of the robot on the upper side of the walking surface.

\section{B. Impact Model}

In the case of a stiff-legged robot on a flat surface, the notion of the contact point of the swing leg with the walking surface would appear to be physically ambiguous, since, without a knee, and with equal length legs, the swing leg must scuff along the ground if it remains in the saggital plane. McGeer [38] has shown with his ballistic walkers, both theoretically and experimentally, that one can basically ignore the leg clearance issue for stiff-legged models. He has done this in two ways: in one realization, he puts additional small motors on the legs that allow him to push the swing leg just slightly out of the saggital plane during the swing phase and to pull the leg back into the saggital plane whenever he wishes to initiate contact. The second way he has done this is to put small (essentially massless) flaps on the ends of the legs, and to fold up the flap of the swing leg during the swing phase, and to unfold it whenever he wants to initiate contact. With McGeer's first method in mind, it is hereafter assumed that contact is initiated when the angle of the stance leg attains a desired value, $\theta_{1}^{d}$. In order for the lengths of the legs to be equal at contact, it must be the case that $\theta_{2}=-\theta_{1}$ at contact. This will be taken care of in the control design of Section IV.

The impact between the swing leg and the ground is modeled as a contact between two rigid bodies. There are many rigid impact models in the literature [2], [7], [8], [28], and [44], and under reasonable hypotheses all of them can be used to obtain an expression for the velocity of the generalized coordinates after the impact of the swing leg with the walking surface in terms of the velocity and position before the impact. The model from [28] is used here. The motion of the robot is only analyzed for the case that the contact of the swing leg with the ground results in no rebound and no slipping of the swing leg, and the stance leg naturally lifting from the ground without interaction [28]. The conditions for these assumptions to be valid will be indicated.

The contact model requires the full five degrees of freedom of the robot. Add Cartesian coordinates $\left(z_{1}, z_{2}\right)^{\prime}$ to the end of the stance leg, as indicated in Fig. 1. This gives once again a model of the form

$$
D_{e}\left(q_{e}\right) \ddot{q}_{e}+C_{e}\left(q_{e}, \dot{q}_{e}\right) \dot{q}_{e}+G_{e}(q)=B_{e}\left(q_{e}\right) u+\delta F_{\mathrm{ext}}
$$

where $q_{e}=\left(\theta_{1}, \theta_{2}, \theta_{3}, z_{1}, z_{2}\right)^{\prime}$ is the set of generalized coordinates and $\delta F^{\text {ext }}$ represents the external forces acting on the robot at the contact point(s). The basic premises in [28] are that: 1) the impact takes place over an infinitesimally small period of time; 2) the external forces during the impact can be represented by impulses; 3 ) impulsive forces may result in an instantaneous change in the velocities of the generalized coordinates, but the positions remain continuous; and 4) the torques supplied by the actuators are not impulsional. With these assumptions, (3) is "integrated" over the "duration" of the impact to obtain [28]

$$
D_{e}\left(q_{e}\right)\left(\dot{q}_{e}^{+}-\dot{q}_{e}^{-}\right)=F^{\mathrm{ext}}
$$

where $F^{\text {ext }}:=\int_{t^{-}}^{t^{+}} \delta F^{\operatorname{ext}}(\tau) d \tau$ is the result of integrating the contact impulse over the impact duration, $\dot{q}_{e}^{+}$is the velocity just after the impact and $\dot{q}_{e}^{-}$is the velocity just before the impact (a more rigorous treatment of this can be found in [8]). Since the positions do not change during the impact, $q_{e}^{+}=q_{e}^{-}$.

In order to be able to solve for all of the unknowns, the above equations must be augmented with additional equations that proscribe what happens at the two contact ends. According to [28], since the stance leg is assumed to detach from the ground without interaction, the external forces acting at the pivot point are zero. Thus $F^{\text {ext }}$ need only consider the external forces at the end of the swing leg. To compute it, let $\Upsilon$ denote the Cartesian-coordinates of the end of the swing leg:

$$
\Upsilon\left(q_{e}\right):=\left[\begin{array}{c}
z_{1}+r \sin \left(\theta_{1}\right)-r \sin \left(\theta_{2}\right) \\
z_{2}+r \cos \left(\theta_{1}\right)-r \cos \left(\theta_{2}\right)
\end{array}\right] .
$$

Then,

$$
F^{\mathrm{ext}}=E^{\prime}\left[\begin{array}{c}
F_{T} \\
F_{N}
\end{array}\right]
$$

where

$$
E:=\frac{\partial \Upsilon}{\partial q_{e}}=\left[\begin{array}{ccccc}
r \cos \left(\theta_{1}\right) & -r \cos \left(\theta_{2}\right) & 0 & 1 & 0 \\
-r \sin \left(\theta_{1}\right) & r \sin \left(\theta_{2}\right) & 0 & 0 & 1
\end{array}\right]
$$

and $F_{T}, F_{N}$ are the tangent and normal forces, respectively, applied at the end of the swing leg.

Equation (4) thus represents five equations and seven unknowns. The unknowns are $\dot{q}_{e}^{+}$and $F^{\text {ext }} ; \dot{q}_{e}^{-}$is known since it equals $\left(\omega_{1}^{-}, \omega_{2}^{-}, \omega_{3}^{-}, \dot{z}_{1}^{-}, \dot{z}_{2}^{-}\right)^{\prime}$, where $\dot{x}^{-}=0$ and $\dot{z}^{-}=0$ since the stance leg acts as a pivot before impact. An additional set of two equations is obtained from the condition that the swing leg does not rebound nor slip at impact, namely, $(d / d t) \Upsilon\left(q_{e}\right)=$ $\left(\partial \Upsilon / \partial q_{e}\right) \dot{q}_{e}^{+}=0$; that is,

$$
E \dot{q}_{e}^{+}=0 .
$$


Equations (4) and (8) are linear in the unknowns and can be solved for $\dot{q}_{e}^{+}, F_{T}$ and $F_{N}$. In Appendix A, it is verified that a unique solution always exists. The result of solving (4) and (8) yields an expression for $\dot{q}_{e}^{+}$in term of $\dot{q}_{e}^{-}$, which should then be used to re-initialize the model (2). In order to do this, a change of coordinates is necessary since the former swing leg is now in contact with the ground, while (1) and (2) assume that $\theta_{1}$ parameterizes the stance leg. The final result is an expression for $x^{+}:=\left(\theta^{+}, \omega^{+}\right)$in terms of $x^{-}:=\left(\theta^{-}, \omega^{-}\right)$, which is written as

$$
x^{+}=\Delta\left(x^{-}\right)
$$

The function $\Delta$ is given in Appendix A. It is also proven in Appendix A that $\Delta$ is continuous.

\section{Overall Model: System with Impulse Effects}

The overall biped model can now be expressed as a system with impulse effects. Assume that the system trajectories possess finite left and right limits, and denote them by $x^{-}(t):=\lim _{\tau,{ }_{t}} x(\tau)$ and $x^{+}(t):=\lim _{\tau \backslash t} x(\tau)$, respectively. The model is, then,

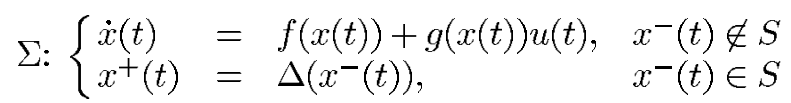

where $S:=\left\{(\theta, \omega) \in \mathcal{X} \mid \theta_{1}=\theta_{1}^{d}\right\}$. The mathematical meaning of a solution of the model will be made precise in Section III. In simple words, a trajectory of the robot is specified by the mechanical model until an impact occurs. Impact occurs when the state "attains" the set $S$, which represents the walking surface. At this point, the impact with the surface results in a very rapid change in the velocity components of the state vector. The impulse model of the impact compresses the impact event into an instantaneous moment in time, resulting in a discontinuity in the velocities. The ultimate result of the impact model is a new initial condition from which the mechanical model evolves until the next impact. In order for the state not to be obliged to take on two values at the "impact time," the impact event is, roughly speaking, described in terms of the values of the state "just prior to impact" at time " $t^{-}$," and "just after impact" at time " $t^{+}$." These values are represented by the left and right limits, $x^{-}$and $x^{+}$, respectively.

For later use, note that $S$ can be expressed as the level set of a function $H: \mathcal{X} \rightarrow \mathbb{R}$. Define $H(x)=\theta_{1}^{d}-\theta_{1}$, so that $S:=\{(\theta, \omega) \in \mathcal{X} \mid H(x)=0\}$. Moreover, it can be easily checked that for each point $s \in S,(\partial H / \partial x)(s) \neq 0$ This implies that $S$ is a smooth embedded submanifold of $\mathcal{X}$ [30].

\section{Method OF PoINCARÉ Sections FOR SYSTEMS WITH IMPULSE EFFECTS}

Nonlinear systems with impulse effects have not been extensively studied. A stability analysis for equilibrium points can be found in [3] and [59], using Lyapunov methods. However, a walking cycle clearly corresponds to a nontrivial periodic orbit, and not to an equilibrium solution of the model, and thus the analysis of [3] and [59] is not applicable. This section contains the definition of a solution of a system with impulse effects, the definition of a periodic orbit, and Lyapunov stability notions for periodic orbits. With these notions in place, the method of Poincaré sections, an important tool for analyzing the stability properties of periodic orbits in ordinary differential equations, is extended to systems with impulse effects. While the basic method carries over nicely to this new setting, the proof differs considerably from the standard one in [43] and [34], for example. In particular, Section IV will need a version ${ }^{3}$ of the Poincaré method that is applicable to continuous, but not Lipschitz continuous, systems. The development will be kept as compact as possible, with all proofs and several lemmas relegated to Appendix B.

\section{A. Basic Definitions}

A function $\varphi:\left[t_{0}, t_{f}\right) \rightarrow \mathcal{X}, t_{f} \in \mathbb{R} \cup\{\infty\}, t_{f}>t_{0}$, is a solution $^{4}$ of (10) if: 1) $\varphi(t)$ is right continuous on $\left.\left[t_{0}, t_{f}\right), 2\right)$ left limits exist at each point of $\left(t_{0}, t_{f}\right)$, and 3$)$ there exists a closed discrete subset $\mathcal{T} \subset\left[t_{0}, t_{f}\right)$ such that: a) for every $t \notin \mathcal{T}, \varphi(t)$ is differentiable and $(d \varphi(t) / d t)=f[\varphi(t)]+g[\varphi(t)] u(t)$ and $\mathrm{b})$ for $t \in \mathcal{T}, \varphi^{-}(t) \in S$ and $\varphi^{+}(t)=\Delta\left[\varphi^{-}(t)\right]$. The condition that the set of impact times is closed and discrete simply means that there is no "chattering" about an impact point. A solution $\varphi(t)$ of (10) is periodic if there exists a finite $T>0$ such that $\varphi(t+T)=\varphi(t)$ for all $t \in\left[t_{0}, \infty\right)$. A set $\mathcal{O} \subset \mathcal{X}$ is a periodic orbit of (10) if $\mathcal{O}=\left\{\varphi(t) \mid t \geq t_{0}\right\}$ for some periodic solution $\varphi(t)$. An orbit is nontrivial if it contains more than one point.

In the following, it is assumed that $u(t)$ in (10) is identically zero, so that one may refer to (10) as being time-invariant. It is further assumed that solutions to (10), when they exist, are unique.

A periodic orbit $\mathcal{O}$ is stable in the sense of Lyapunov if for every $\epsilon>0$, there exists an open neighborhood $\mathcal{V}$ of $\mathcal{O}$ such that for every $p \in \mathcal{V}$, there exists a solution $\varphi:[0, \infty) \rightarrow \mathcal{X}$ of (10) satisfying $\varphi(0)=p$ and $\operatorname{dist}(\varphi(t), \mathcal{O})<\epsilon$ for all $t \geq 0$. $\mathcal{O}$ is $\{$ lit attractive $\}$ if there exists an open neighborhood $\mathcal{V}$ of $\mathcal{O}$ such that for every $p \in \mathcal{V}$, there exists a solution $\varphi:[0, \infty) \rightarrow \mathcal{X}$ of (10) satisfying $\varphi(0)=p$ and $\lim _{t \rightarrow \infty} \operatorname{dist}(\varphi(t), \mathcal{O})=0$. $\mathcal{O}$ is asymptotically stable in the sense of Lyapunov if it is both stable and attractive. From here on, the qualifier, "in the sense of Lyapunov," will be systematically assumed if it is not made explicit.

Finally, assume that in (10), $S=\{x \in \mathcal{X} \mid H(x)=0\}$, where $H: \mathcal{X} \rightarrow \mathbb{R}$ is continuously differentiable. A periodic orbit $\mathcal{O}$ is transversal to $S$ if its closure intersects $S$ in exactly one point, and for $\bar{x}:=\overline{\mathcal{O}} \cap S, L_{f} H(\bar{x}):=(\partial H / \partial x)(\bar{x}) f(\bar{x}) \neq$ 0 (in words, at the intersection, $\overline{\mathcal{O}}$ is not tangent to $S$, where $\overline{\mathcal{O}}$ is the set closure of $\mathcal{O}$ ). In the case of the biped robot, a nontrivial periodic orbit transversal to $S$ will also be referred to as a periodic walking cycle.

1) Remark: Note that a periodic orbit of a system with impulse effects may not be a closed set, since, for $t \in \mathcal{T}, \varphi^{-}(t) \notin$ $\mathcal{O}$ (if solutions were assumed to be left continuous, instead of right continuous, then $\varphi^{+}(t) \notin \mathcal{O}$ ). Indeed, a periodic orbit is

\footnotetext{
${ }^{3}$ The standard development assumes that the flow is a local diffeomorphism, while, here, it will not even be a homeomorphism.

${ }^{4}$ The definition is based on [59], except that solutions are taken to be right continuous instead of left continuous. For a careful study of the existence of solutions of mechanical systems with shocks, see [8] and [55].
} 
closed if, and only if, $\mathcal{T}=\emptyset$. For a biped robot, a closed periodic orbit would not correspond to walking because there would be no impact with the walking surface.

\section{B. Poincaré's Method}

The method of Poincaré sections is extended to systems with impulse effects (10), for the case of nontrivial periodic orbits that are transversal to $S$. This will be done in a certain amount of generality so that a wide class of biped robot models and controllers can be treated. In particular, the finite-time stabilizing controllers of Section IV will require the use of feedbacks that are continuous, but not Lipschitz continuous.

Consider a time-invariant system with impulse effects

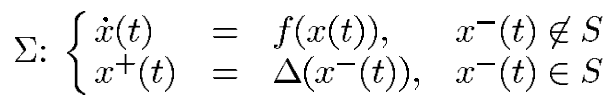

where the state space $\mathcal{X}$ is an open subset of $\mathbb{R}^{n}$. The hypotheses that will be used in its analysis are listed below. As a point of notation, $\varphi$ will be used to denote a solution of the system (11), as defined in Section III-A, and $\varphi^{f}$ will denote a solution of the associated ordinary differential equation,

$$
\dot{x}=f(x) \text {. }
$$

The point of introducing $\varphi^{f}$ is that, firstly, a lot is known about solutions of ordinary differential equations with continuous right-hand sides [24]. Secondly, in view of the first point, it is convenient to prove properties of (11) in term of properties of (12). Finally, at times in the proofs, it is necessary to extend a solution of (12) "through" $S$, while this does not make sense for (11) (that is, for the robot, it does not make sense for its "foot to be stuck in the ground").

\section{1) Hypotheses:}

H1) $f(x)$ is continuous on $\mathcal{X}$.

H2) A solution of (12) from a given initial condition is unique and depends continuously on the initial condition.

H3) There exists a differentiable function $H: \mathcal{X} \rightarrow \mathbb{R}$ such that $S=\{x \in \mathcal{X} \mid H(x)=0\}$. Moreover, for every $s \in S,(\partial H / \partial x)(s) \neq 0$.

H4) $\Delta: S \rightarrow \mathcal{X}$ is continuous, where $S$ is given the subset topology from $\mathcal{X}$.

Hypothesis $H 1$ implies that at any point $x_{0} \in \mathcal{X}$, a solution to (12) will exist over a sufficiently small interval of time [24]. This solution may not be unique, and may not depend continuously on the initial condition, as in H2. Hypothesis $H 3$ implies that $S$ is an embedded submanifold [30], when given the subset topology. Hypothesis $\mathrm{H} 4$ assures that the result of an impact varies continuously with respect to where it occurs on $S$.

The first goal is to define the Poincaré return map. Define the time to impact function, $T_{I}: \mathcal{X} \rightarrow \mathbb{R} \cup\{\infty\}$, by

$$
T_{I}\left(x_{0}\right):= \begin{cases}\inf \left\{t \geq 0 \mid \varphi^{f}\left(t, x_{0}\right) \in S\right\}, & \text { if } \exists t \text { such that } \\ & \varphi^{f}\left(t, x_{0}\right) \in S \\ & \text { otherwise }\end{cases}
$$

From Lemma 3 in Appendix B, Hypotheses $\mathrm{H} 1-\mathrm{H} 3$ imply that $T_{I}$ is continuous at points $x_{0}$ where $0<T_{I}\left(x_{0}\right)<\infty$ and
$L_{f} H\left(\varphi^{f}\left(T_{I}\left(x_{0}\right), x_{0}\right)\right) \neq 0$. Hence, under H1-H3, $\tilde{\mathcal{X}}:=\{x \in$ $\mathcal{X} \mid 0<T_{I}(x)<\infty$ and $\left.L_{f} H\left(\varphi^{f}\left(T_{I}(x), x\right)\right) \neq 0\right\}$ is open. If H4 also holds, then $\widetilde{S}:=\Delta^{-1}(\widehat{\mathcal{X}})$ is an open subset of $S$. It immediately follows that under $\mathrm{H} 1-\mathrm{H} 4$, the Poincaré return map, $P: \widetilde{S} \longrightarrow S$ by

$$
P(x):=\varphi^{f}\left(T_{I}(\Delta(x)), \Delta(x)\right)
$$

is well-defined and continuous. In the case of the robot, the return map represents the evolution of the robot just before an impact with the walking surface, to just before the next impact, assuming that next impact does occur. If it does not, that is, the robot falls due to the preceeding impact, the point being analyzed is not in the domain of definition of the return map.

$\mathrm{Next}$, note that under $\mathrm{H} 1-\mathrm{H} 4$, if $\mathcal{O}$ is any periodic orbit of (11) that is transversal to $S$, then $\mathcal{O} \subset \tilde{\mathcal{X}}$. This is essentially by definition. Thus, there exists $x_{0} \in \widetilde{S}$ that generates $\mathcal{O}$ in the sense that $\Delta\left(x_{0}\right) \in \mathcal{O}$; indeed, $x_{0}=\overline{\mathcal{O}} \cap \tilde{S}$. It thus makes sense to denote the orbit by $\mathcal{O}\left(\Delta\left(x_{0}\right)\right)$.

Theorem 1(Method of Poincaré Sections for Systems with Impulse Effects): Under H1-H4, the following statements hold.

a) If $\mathcal{O}$ is a periodic orbit of (11) that is transversal to $S$, then there exists a point $x_{0} \in \tilde{S}$ that generates $\mathcal{O}$.

b) $x_{0} \in \widetilde{S}$ is a fixed point of $P$ if, and only if, $\Delta\left(x_{0}\right)$ generates a periodic orbit that is transversal to $S$.

c) $x_{0} \in \widetilde{S}$ is a stable equilibrium point of $x_{k+1}=P\left(x_{k}\right)$ if, and only if, the orbit $\mathcal{O}\left(\Delta\left(x_{0}\right)\right)$ is stable in the sense of Lyapunov.

d) $x_{0} \in \tilde{S}$ is an asymptotically stable equilibrium point of $x_{k+1}=P\left(x_{k}\right)$ if, and only if, the orbit $\mathcal{O}\left(\Delta\left(x_{0}\right)\right)$ is asymptotically stable in the sense of Lyapunov.

The proof of the theorem is given in Appendix B.

\section{Asymptotically Stable Walking}

This section develops a feedback controller for the system with impulse effects, (10), in the particular case of the biped robot given by the differential equation (2) and the impact model (9). The goal of the control design is to induce an asymptotically stable walking cycle, and to facilitate the verification of its existence and stability properties. The verification will be done using the method of Poincaré.

\section{A. Encoding a Walking Pattern}

At its most basic level, walking consists of two things [45]: posture control, that is, maintaining the torso in a semi-erect position, and swing leg advancement, that is, causing the swing leg to come from behind the stance leg, pass it by a certain amount, and prepare for contact with the ground. This motivates the direct control of the angles $\theta_{3}$ (describing the torso) and $\theta_{2}$ (describing the swing leg). As discussed in Section I, the most common approach to control in the multi-ped literature is through the tracking of precomputed reference trajectories. That is, in the context of the robot model investigated here, the first step of the control design would be to determine functions of time $\theta_{2}(t)$ and $\theta_{3}(t)$ that express a desired behavior of the robot. Then, standard control techniques would be employed to induce "asymptotic" tracking of these trajectories. The resulting closed-loop system is nonlinear, hybrid (due to the impact map), 
time-varying (due to the time-dependent reference trajectories) and very difficult to analyze.

On a periodic orbit corresponding to a normal walking motion, it is clear that the horizontal motion of the hips is monotonically strictly increasing. For the biped of Fig. 1, this is equivalent to $\theta_{1}(t)$ strictly increasing over each step of the walking cycle. Thus, for any desired trajectories $\theta_{2}(t)$ and $\theta_{3}(t)$ that express (encode) a desired walking pattern for the biped, it is therefore reasonable to assume that the corresponding trajectory for $\theta_{1}$ has the property that $\theta_{1}(t)$ is strictly monotonic. It follows that $\theta_{2}(t)$ and $\theta_{3}(t)$ can each be re-parameterized in terms of $\theta_{1}$. That is, without loss of generality, it can be supposed that $\theta_{3}(t)=\eta_{1}\left(\theta_{1}(t)\right)$ and $\theta_{2}(t)=\eta_{2}\left(\theta_{1}(t)\right)$, for some functions $\eta_{i}$.

The simplest version of posture control is to maintain the angle of the torso at some constant value, say $\theta_{3}^{d}$, while the simplest version of swing leg advancement is to command the swing leg to behave as the mirror image of the stance leg, that is, $\theta_{2}=-\theta_{1}$. Thus the "behavior" of walking will be "encoded" into the dynamics of the robot by defining outputs

$$
y:=\left[\begin{array}{l}
y_{1} \\
y_{2}
\end{array}\right]:=\left[\begin{array}{l}
h_{1}(\theta) \\
h_{2}(\theta)
\end{array}\right]:=\left[\begin{array}{c}
\theta_{3}-\eta_{1}\left(\theta_{1}\right) \\
\theta_{2}-\eta_{2}\left(\theta_{1}\right)
\end{array}\right]:=\left[\begin{array}{c}
\theta_{3}-\theta_{3}^{d} \\
\theta_{2}+\theta_{1}
\end{array}\right]
$$

with the control objective being to drive the outputs to zero. Driving $y$ to zero will force $\theta_{2}$ and $\theta_{3}$ to converge to known functions of $\theta_{1}$ (here, $\theta_{3}^{d}$, being a constant, should be viewed as a trivial function of $\theta_{1}$ ). This will be one of the key steps in reducing the stability analysis problem to that of a map from $\mathbb{R}$ to $\mathbb{R}$.

Of course, the idea of building in a dynamic behavior of a system through the judicious definition of a set of outputs, which when nulled yields a desirable internal behavior, is not novel in control [30] nor walking robots [32], [26], [9], [31], [40], [51], [16]. However, it is interesting to note that this idea, which seems to be an essential step for proving anything about the trajectories of the closed-loop system, has been best used to analytical advantage in the monoped (one-legged hopper) literature. This seems to be due to the fact that accurate, approximate, analytically tractable models of the hopper exist, and the associated Poincaré return map can be analyzed in considerable detail [9], [51], [16]. This has led to the determination of sampled-data control laws (sampling is done synchronously with impact events) that lead to explicit, low-dimensional tests for asymptotic stability of a periodic orbit.

\section{B. Controller Design}

Since the system (2) comes from the second order model (1), and the outputs (15) only depend upon $\theta$, it follows that the relative degree of each output component is either two or infinite. Direct computation gives that [41]

$$
\ddot{y}=L_{f}^{2} h(x)+L_{g} L_{f} h(x) u
$$

and that the determinant of the decoupling matrix, $L_{g} L_{f} h$, is [see Appendix A, (51)] is zero if, and only if,

$$
-r\left(r M_{H}+r m+r M_{T}+l M_{T} \cos \left(\theta_{1}-\theta_{3}\right)\right)=0 .
$$

Thus, the decoupling matrix is invertible for all $x \in \mathcal{X}$ as long as $0<l M_{T}<r\left(m+M_{T}+M_{H}\right)$, which imposes a very mild constraint on the position of the center of gravity of the upper body of the robot in relation to the length of its legs. This leads to the following hypothesis.

\section{1) Hypothesis:}

CH1) The decoupling matrix is globally invertible.

From now on, it is supposed that $\mathrm{CH} 1$ is met. Therefore, due to the global invertibility of the decoupling matrix, stabilizing dynamics for the output of system (2) can be assigned. The easiest way to do this is to first decouple the system, [30], [41], [37] and then impose a desired dynamic response. In preparation for doing this, note that $\Phi: M \rightarrow \mathbb{R}^{3}$ by

$$
\Phi(\theta):=\left[\begin{array}{l}
y_{1} \\
y_{2} \\
\theta_{1}
\end{array}\right]=\left[\begin{array}{c}
\theta_{3}-\theta_{3}^{d} \\
\theta_{1}+\theta_{2} \\
\theta_{1}
\end{array}\right]
$$

is a diffeomorphism onto its range. With this coordinate transformation, and upon defining

$$
v:=L_{f}^{2} h+L_{g} L_{f} h u
$$

the system can be written in the decoupled form

$$
\left[\begin{array}{c}
\ddot{y} \\
\ddot{\theta}_{1}
\end{array}\right]=\left[\zeta_{0}\left(y, \dot{y}, \theta_{1}, \dot{\theta}_{1}\right)+\zeta_{1}\left(y, \dot{y}, \theta_{1}, \dot{\theta}_{1}\right) v\right] \text {. }
$$

The next step is to impose a continuous feedback $v=v(y, \dot{y})$ on (19), and thus on (10), so that the pair of double integrators $\ddot{y}=v$ is globally finite-time stabilized [23], [4]-[6]. This will collapse the image of the Poincaré return map to a one-dimensional set.

2) Hypotheses: The closed-loop pair of double integrators, $\ddot{y}=v(y, \dot{y})$, satisfies the following conditions.

$\mathrm{CH} 2$ ) Solutions globally exist on $\mathbb{R}^{4}$, and are unique.

CH3) Solutions depend continuously on the initial conditions.

CH4) The origin is globally asymptotically stable, and convergence is achieved in finite time.

CH5) The settling time function, ${ }^{5} T_{\text {set }}: \mathbb{R}^{4} \rightarrow \mathbb{R}$ by

$$
\begin{aligned}
T_{\text {set }}\left(y_{0}, \dot{y}_{0}\right):= & \inf \{t>0 \mid(y(t), \dot{y}(t))=(0,0), \\
& (y(0), \dot{y}(0))=\left(y_{0}, \dot{y}_{0}\right)
\end{aligned}
$$

depends continuously on the initial condition, $\left(y_{0}, \dot{y}_{0}\right)$.

Hypotheses $\mathrm{CH} 2-\mathrm{CH} 4$ correspond to the definition of finite-time stability [23], [4]; CH5 will also be needed, but is not implied by $\mathrm{CH} 2-\mathrm{CH} 4$ [5]. These requirements rule out traditional sliding mode control, with its well-known discontinuous action. A means of meeting these four objectives can be found in [4], [5]. The first two parts of the following lemma are proven in [4]. The continuity of the settling time function is proven in [5] (a continuous upper bound on the settling time function is given in [4], along with a Lyapunov function).

\footnotetext{
${ }^{5}$ That is, the time it takes for a solution initialized at $\left(y_{0}, \dot{y}_{0}\right)$ to converge to the origin. The terminology is taken from [4].
} 
Lemma 1: [Bhat and Bernstein] Consider the double integrator on $\mathbb{R}^{2}$

$$
\begin{aligned}
& \dot{x}_{1}=x_{2} \\
& \dot{x}_{2}=\nu .
\end{aligned}
$$

with scalar input $\nu$. Then, for all $0<\alpha<1$, the feedback

$$
\begin{aligned}
\nu & =\psi_{\alpha}\left(x_{1}, x_{2}\right) \\
& :=-\operatorname{sign}\left(x_{2}\right)\left|x_{2}\right|^{\alpha}-\operatorname{sign}\left(\phi_{\alpha}\left(x_{1}, x_{2}\right)\right)\left|\phi_{\alpha}\left(x_{1}, x_{2}\right)\right|^{\alpha / 2-\alpha},
\end{aligned}
$$

where $\phi_{\alpha}\left(x_{1}, x_{2}\right):=x_{1}+(1 / 2-\alpha) \operatorname{sign}\left(x_{2}\right)\left|x_{2}\right|^{2-\alpha}$, satisfies the following.

P1) $\quad \nu$ is continuous.

P2) The origin of (20) in closed loop with (21) is globally finite-time stable.

P3) The settling time function, $T_{\text {set }}$, depends continuously on the initial condition.

Let $\bar{\psi}^{i}\left(x_{1}, x_{2}\right), i=1,2$, be any feedbacks for (20) meeting P1-P3 of Lemma 1. To each double integrator of (19), apply the feedback $v_{i}=\bar{\psi}^{i}\left(y_{i}, \dot{y}_{i}\right)$, so that, with

$$
v:=\Psi(y, \dot{y}):=\left[\begin{array}{l}
\bar{\psi}^{1}\left(y_{1}, \dot{y}_{1}\right) \\
\bar{\psi}^{2}\left(y_{2}, \dot{y}_{2}\right)
\end{array}\right]
$$

$\mathrm{CH} 2-\mathrm{CH} 5$ are satisfied for $\ddot{y}=v$. Define a feedback on (2), and hence on (10) as well, by

$$
u(x):=\left(L_{g} L_{f} h(x)\right)^{-1}\left(\Psi\left(h(x), L_{f} h(x)\right)-L_{f}^{2} h(x)\right)
$$

and denote the right-hand side of the closed loop by

$$
f_{c l}(x):=f(x)+g(x) u(x) .
$$

Finally, define

$$
T_{\text {set }}^{c l}(x):=\max \left\{T_{\text {set }}\left(h_{1}, L_{f} h_{1}\right), T_{\text {set }}\left(h_{2}, L_{f} h_{2}\right)\right\}
$$

in the obvious way. It follows that $T_{\text {set }}^{c l}(x)$ is a continuous function of $x$.

The model of the biped robot in closed loop with the controller is

$$
\Sigma_{c l}:\left\{\begin{aligned}
\dot{x}(t) & =f_{c l}(x(t)), & & x^{-}(t) \notin S \\
x^{+}(t) & =\Delta\left(x^{-}(t)\right), & & x^{-}(t) \in S .
\end{aligned}\right.
$$

In the next section, the method of Poincare sections will be applied to analyze the existence and stability of periodic orbits. The finite-time convergence property of the controller will be exploited to deduce properties of the solutions of (26) by studying the solutions of

$$
\dot{x}(t)=f_{c l}(x(t))
$$

corresponding to a one-dimensional subset of initial conditions.

\section{Analysis à la Poincaré}

The first step in the analysis is to verify that Hypotheses H1-H4 hold for the closed-loop system (26). Lemma 5 of Appendix B shows that continuity of the feedback (22) plus Hypotheses $\mathrm{CH} 1-\mathrm{CH} 3$ imply $\mathrm{H} 1$ and $\mathrm{H} 2$. Hypotheses $\mathrm{H} 3$ and $\mathrm{H} 4$ were verified in Section II-C and Section II-B, respectively. Thus Theorem 1 is applicable. The second step in the analysis is to simplify the application of the theorem. This is achieved by studying the image of the Poincare return map in the case that the controller has had sufficient time to converge. Convergence of the controller is equivalent to the outputs (15) being identically zero over an interval of time.

The internal dynamics of the system (2) compatible with the output (15) being identically zero is called the zero dynamics [30], and the state space on which the zero dynamics evolves is called the zero dynamics manifold. For the biped model under study, the zero dynamics manifold is computed from (19) to be

$$
\begin{gathered}
Z=\left\{(\theta, \omega) \in \mathcal{X} \mid \theta_{3}=\theta_{3}^{d}, \theta_{1}+\theta_{2}=0, \omega_{3}=0,\right. \\
\left.\omega_{1}+\omega_{2}=0,-\pi<\theta_{1}<\pi, \omega_{1} \in \mathbb{R}\right\} .
\end{gathered}
$$

Note that the feedback (23) makes $Z$ an invariant manifold of (2), while the same feedback does not render $Z$ invariant for (10) since $\Delta$ does not map $Z \cap S$ into $Z$. The zero dynamics itself will not be computed here since it is not needed directly in the stability analysis; the zero-dynamics will be studied in Section $\mathrm{V}$ (see also Appendix A).

Lemma 2: Under Hypotheses $\mathrm{CH} 1-\mathrm{CH} 5$, and $\mathrm{H} 3-\mathrm{H} 4$, the following apply.

1) The set

$$
\begin{aligned}
\hat{S}:= & \left\{x_{0} \in S \mid T_{\mathrm{set}}\left(x_{0}\right)<T_{I}\left(x_{0}\right)<\infty,\right. \\
& \left.L_{f} H\left(\phi^{f}\left(T_{I}\left(x_{0}\right), x_{0}\right)\right) \neq 0\right\}
\end{aligned}
$$

is an open subset of $\widetilde{S}$.

2) Let $P: \widetilde{S} \rightarrow S$ be the Poincaré return map. Then $P: \hat{S} \rightarrow$ $S \cap Z$.

The straightforward proof is skipped. Note that in terms of the original coordinates $(\theta, \omega)$ of the robot,

$$
\begin{aligned}
S \cap Z=\left\{(\theta, \omega) \in \mathcal{X} \mid \theta_{3}=\right. & \theta_{3}^{d}, \theta_{1}+\theta_{2}=0, \omega_{3}=0, \\
& \left.\omega_{1}+\omega_{2}=0, \theta_{1}=\theta_{1}^{d}, \omega_{1} \in \mathbb{R}\right\},
\end{aligned}
$$

a one-dimensional (embedded) submanifold of $\mathcal{X}$. Define

$$
\rho: \hat{S} \cap Z \rightarrow S \cap Z \text { by } \rho(x):=P(x) \text {. }
$$

For $x^{*} \in \hat{S}, P\left(x^{*}\right) \in S \cap Z$. Thus, by the definition of $\rho$, $P\left(x^{*}\right)=x^{*}$ if, and only if, $x^{*} \in \hat{S} \cap Z$ and $\rho\left(x^{*}\right)=x^{*}$. Suppose that for some $x_{0} \in \hat{S}$, the sequence $x_{k+1}:=P\left(x_{k}\right)$ is well-defined for $k \geq 0$, and remains in some open neighborhood of $x_{0}$. Then for all $\bar{k} \geq 1, x_{k+1}=\rho\left(x_{k}\right)$. It follows that $x^{*} \in \hat{S}$ is a stable (resp., asymptotically stable) equilibrium point of $P$ if, and only if, it is a stable (resp., asymptotically stable) equilibrium point of $\rho$. Thus, the determination of the existence and stability properties of periodic orbits that are transversal to $\hat{S}$ can be reduced to the analysis of a one-dimensional map. These results are summarized in the following theorem. A numerical example to the biped robot is given immediately in the next subsection.

Theorem 2(Method of Poincaré for Finite-Time Control): Consider the biped robot model of Section II, written in the form of a system with impulse effects, (10). Define outputs such that Hypothesis $\mathrm{CH} 1$ is met. Suppose that a continuous, finite-time 
stabilizing feedback is applied, and that Hypotheses $\mathrm{CH} 2-\mathrm{CH} 4$ are met. Define $Z, \hat{S}$, and $\rho$ as in (28), (29) and (30), respectively. Then,

1) a periodic orbit is transversal to $\hat{S}$ if, and only if, it is transversal to $\hat{S} \cap Z$;

2) $x^{*} \in \hat{S} \cap Z$ gives rise to a periodic orbit of (26) if, and only if, $\rho\left(x^{*}\right)=x^{*}$;

3) $x^{*} \in \hat{S} \cap Z$ gives rise to a stable (resp., asymptotically stable) periodic orbit of (26) if, and only if, $x^{*}$ is a stable (resp., asymptotically stable) equilibrium point of $\rho$.

\section{Numerical Example}

Consider the model (10), with the following values of the parameters:

$$
m=5 \quad M_{H}=15 \quad M_{T}=10 \quad r=1 \quad l=0.5
$$

corresponding to the mass of the legs, the mass of the hips, the mass of the torso, the length of the legs and the distance between the center of mass of the hips and the center of mass of the torso. The units are kilograms and meters. With the outputs defined as in (15), Hypothesis $\mathrm{CHI}$ is met. Suppose that the desired inclination angle of the torso is $\theta_{3}^{d}=\pi / 6$ and that the swing leg has been designed to re-enter the saggital plane when $\theta_{1}^{d}=\pi / 8$. In the feedback (23), suppose that

$$
\Psi(x):=\left[\begin{array}{l}
\frac{1}{\epsilon^{2}} \psi_{\alpha}\left(y_{1}, \epsilon \dot{y}_{1}\right) \\
\frac{1}{\epsilon^{2}} \psi_{\alpha}\left(y_{2}, \epsilon \dot{y}_{2}\right)
\end{array}\right]
$$

is used, with $\epsilon=0.1$ and $\alpha=0.9$, where $\psi_{\alpha}\left(x_{1}, x_{2}\right)$ is given by (21). The parameter $\epsilon>0$ allows the settling time of the controller to be adjusted. With this feedback, $\mathrm{CH} 2-\mathrm{CH} 5$ hold. In the impact model (9), it is supposed that the friction coefficient $\mu \geq 2 / 3$ (see Appendix A). In the course of the simulations, it has been verified that the impact model is valid, so this point will not be discussed further.

To determine if this choice of parameters results in an asymptotically stable walking cycle that is transversal to $\hat{S}$, that is, the orbit is transversal to $S$ and the finite-time stabilizing feedback has had enough time to converge over the walking cycle, the function $\rho$ of Theorem 2 must evaluated. This is conveniently done as follows. Define $\sigma: \mathbb{R} \rightarrow \hat{S} \cap Z$ by $\sigma\left(\omega_{1}^{-}\right):=$ $\left(\theta_{1}^{d},-\theta_{1}^{d}, \theta_{3}^{d}, \omega_{1}^{-},-\omega_{1}^{-}, 0\right)$, where $\omega_{1}^{-}$denotes the angular velocity of the stance leg just before impact. Define $\lambda:=\sigma^{-1} \circ$ $\rho \circ \sigma$. A straightforward procedure for evaluating $\lambda$ on the basis of a simulation model ${ }^{6}$ of the closed-loop system is now given.

1) Numerical Procedure to Test for Walking Cycles via the Method of Poincaré:

1) For a point $\omega_{1}^{-}>0$, compute $x^{-}:=\sigma\left(\omega_{1}^{-}\right)$, the position of the robot just before impact (the restriction to positive velocities corresponds to the robot walking from left to right).

\footnotetext{
${ }^{6}$ The existence and continuity of $\lambda$ has been assured by the theoretical developments of the paper. A numerical simulator is being used to compute an approximation of this function. Since the feedback in (22) can be uniformly approximated by a Lipschitz continuous function, a standard numerical integrator can be used to approximately compute $\lambda$ to any desired degree of accuracy.
}
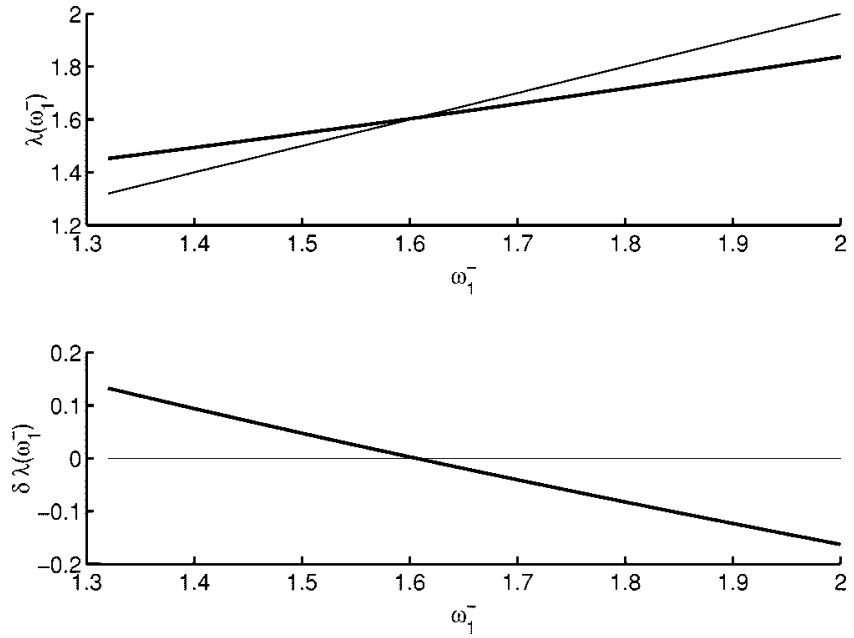

Fig. 2. The top graph presents the function $\lambda$ (bold line) and, for visualization purposes, the identity function (thin line); the bottom graph presents the function $\delta \lambda$ (bold line) and the zero line (thin line). From either graph, it is seen that there exists a periodic orbit and that it is asymptotically stable.

2) Apply the impact model to $x^{-}$, that is, compute $x^{+}:=$ $\Delta\left(x^{-}\right)$

3) Use $x^{+}$as the initial condition in (27), the robot in closed loop with the controller, and simulate until one of the following happens:

a) there exists a time $T>0$ where $\theta_{1}(T)=\theta_{1}^{d}$; then, if $T$ is greater than the settling time of the controller (in other words, the output $y$ is identically zero), then $x^{+} \in \hat{S} \cap Z$, and $\lambda\left(\omega_{1}^{-}\right)=\omega_{1}(T)$; else, $x^{+} \notin \hat{S} \cap Z$, and $\lambda\left(\omega_{1}^{-}\right)$is undefined at this point.

b) there does not exist a $T>0$ such that $\theta_{1}(T)=$ $\theta_{1}^{d}$ (which is normally detected by one of the angles exceeding $\pm \pi / 2$ during the simulation); in this case, it is also true that $x^{+} \notin \hat{S} \cap Z$, and $\lambda\left(\omega_{1}^{-}\right)$ is undefined at this point.

Fig. 2 displays the function $\lambda$; it also displays the related function $\delta \lambda\left(\omega_{1}^{-}\right):=\lambda\left(\omega_{1}^{-}\right)-\omega_{1}^{-}$, which represents the change in velocity over successive cycles, from just before an impact to just before the next one. It is seen that $\lambda$ is undefined for $\omega_{1}^{-}$ less than approximately $1.32 \mathrm{rad} / \mathrm{s}$ (for initial $\omega_{1}^{-}$less than this value, the robot fell backward). The plot was truncated at $2 \mathrm{rad} / \mathrm{s}$ because nothing interesting occurs beyond this point (except an upper bound on its domain of existence will eventually occur due to the controller not having enough time to settle over one walking cycle). A fixed point occurs at approximately 1.6 radians/second, and, from the graph of $\lambda$, it clearly corresponds to an asymptotically stable walking cycle, whose projection is shown in Fig. 3.

To illustrate the role played by the inclination of the torso, suppose that $\theta_{3}^{d}$ is reduced by half to $\pi / 12$. Fig. 4 displays $\lambda$ and $\delta \lambda$ for this case. It is seen that there is no fixed point, and hence no periodic orbit that is transversal to $\hat{S}$. Simulations also support this conclusion, but are not reported here for reasons of space. For a robot without knees or ankles, the driving force for walking comes from the inclination of the torso, which couples in the force of gravity. 


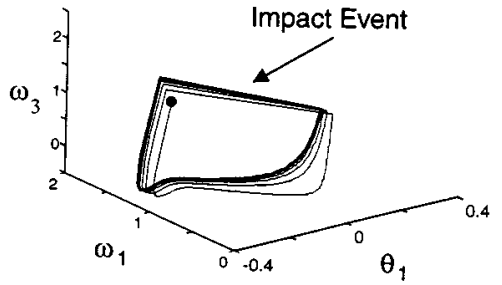

Fig. 3. Projection onto $\left(\theta_{1}, \omega_{1}, \omega_{3}\right)$ of a trajectory asymptotically converging to an orbit. Note that the "flat" portion of the curve is really an instantaneous transition due to the impact of the swing leg with the ground. The dot is the initial point.
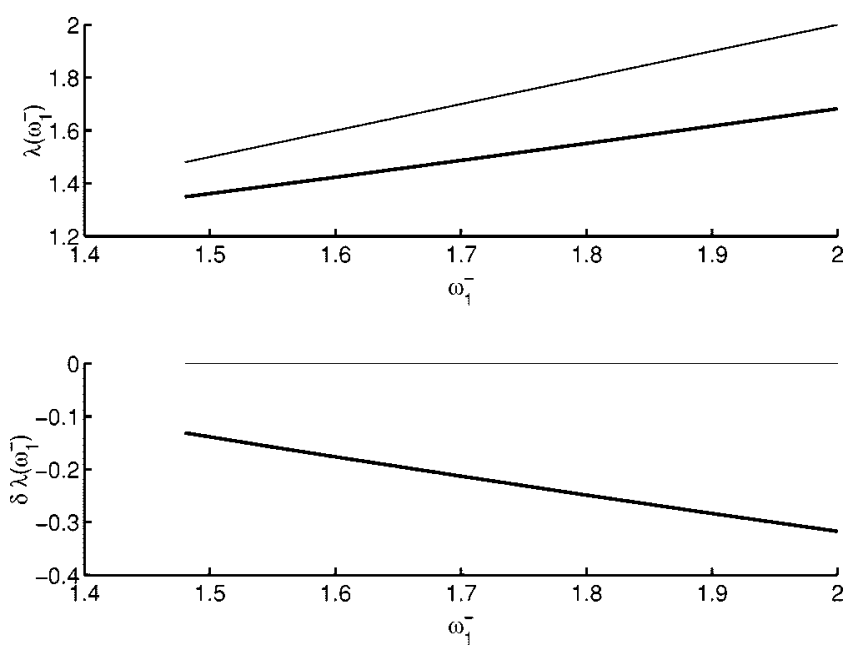

Fig. 4. The top graph presents the function $\lambda$ (bold line) and, for visualization purposes, the identity function (thin line); the bottom graph presents the function $\delta \lambda$ (bold line) and the zero line (thin line). From either graph, it is seen that there does not exist a periodic orbit transversal to $\hat{S}$.

In [22], it is shown that the stability characterization developed in this paper is sufficiently tractable that it can be efficiently incorporated into a (numerical) feedback design scheme for computing an asymptotically stabilizing feedback controller with low peak torque demands and good efficiency with respect to average energy consumption over a cycle, or to optimize the mechanical parameters of the robot itself.

\section{ANALysis of the ZeRo DyNAMics IN RELATION TO HIGH GAIN CONTROL}

The previous sections have provided an effective method for determining the existence of a periodic orbit, and for analyzing its stability properties. The goal of this section is to analyze more deeply the internal behavior of the robot model in closed loop with a finite-time stabilizing controller. As pointed out in Section IV-C, the classical zero dynamics of the mechanical part of the robot model are not invariant under the impact model, when bounded control gains are used. It is shown here that in the limit as the gain tends to infinity, the invariance of the zero dynamics is recovered, independent of the impact model. This can be used to explain certain properties of the Poincaré map, $\lambda$, such as its observed strict monotonicity. For reasons of space, the exposition will be more terse than that of the previous sections.

\section{A. Zero Dynamics}

It is easy to verify that the input vector fields of (2) commute; that is, their Lie bracket is zero. This, in combination with the decoupling matrix being globally invertible, implies that the dynamic (2), with outputs (15), can be transformed into a particularly simple normal form [30]. An appropriate coordinate transformation can be found by applying [30, Proposition 1.3, p. 237], plus the constructive proof of the [30, Frobenius Theorem, p. 26], in this same reference. The result is the following change of coordinates, which is a global diffeomorphism under Hypothesis CHI:

$$
\bar{x}=\left[\begin{array}{llllll}
\theta_{3}-\theta_{3}^{d} & \theta_{1}+\theta_{2} & \omega_{3} & \omega_{1}+\omega_{2} & \theta_{1} & \gamma(x)
\end{array}\right]^{\prime},
$$

where,

$$
\begin{aligned}
\gamma(x)= & \left(\frac{5}{4} m r^{2}+M_{H} r^{2}+M_{T} r^{2}-\frac{1}{2} m r^{2} \cos \left(\theta_{1}-\theta_{2}\right)\right. \\
& \left.+M_{T} r l \cos \left(\theta_{1}-\theta_{3}\right)\right) \omega_{1} \\
& +\left(\frac{1}{4} m r^{2}-\frac{1}{2} m r^{2} \cos \left(\theta_{1}-\theta_{2}\right)\right) \omega_{2} \\
& +\left(M_{T} l^{2}+M_{T} r l \cos \left(\theta_{1}-\theta_{3}\right)\right) \omega_{3}
\end{aligned}
$$

The constructive proof of the Frobenius Theorem shows, in fact, that the function $\gamma$ is the last row of the matrix

$$
\left[B,\left[\begin{array}{l}
0 \\
0 \\
1
\end{array}\right]\right]^{-1} \cdot D \cdot\left[\begin{array}{l}
\omega_{1} \\
\omega_{2} \\
\omega_{3}
\end{array}\right] .
$$

Note that $\left(\bar{x}_{1}, \bar{x}_{2}, \bar{x}_{3}, \bar{x}_{4}\right)=\left(h_{1}(x), h_{2}(x), L_{f} h_{1}(x), L_{f} h_{2}(x)\right)$.

In the $\bar{x}$-coordinates, the state-space model of the robot (2) with the decoupling feedback (18) becomes

$$
\dot{\bar{x}}=\bar{f}(\bar{x}))
$$

where

$$
\bar{f}_{1}=\bar{x}_{3}, \bar{f}_{2}=\bar{x}_{4}, \bar{f}_{3}=v_{1}, \bar{f}_{4}=v_{2}
$$

$$
\begin{aligned}
\bar{f}_{5}= & \frac{4 M_{T} l\left(r \cos \left(\bar{x}_{1}-\bar{x}_{5}+\theta_{3}^{d}\right)-l\right) \bar{x}_{3}+}{4 r\left(m r+M_{H} r+M_{T} r+M_{T} l \cos \left(\bar{x}_{1}-\bar{x}_{5}+\theta_{3}^{d}\right)\right)} \\
\bar{f}_{6}= & g\left(M_{T} l \sin \left(\bar{x}_{1}+\theta_{3}^{d}\right)-\frac{1}{2} m r \sin \left(\bar{x}_{2}-\bar{x}_{5}\right)\right. \\
& \left.+\left(\frac{3}{2} m r+M_{T} r+M_{H} r\right) \sin \left(\bar{x}_{\tilde{5}}\right)\right)
\end{aligned}
$$

The zero dynamics is obtained by imposing $y(t) \equiv 0$. Setting $\left(\bar{x}_{1}, \bar{x}_{2}, \bar{x}_{3}, \bar{x}_{4}\right)=(0,0,0,0)$ in (34), and relabeling $\bar{x}_{5}$ and $\bar{x}_{6}$ by $\xi_{1}$ and $\xi_{2}$, respectively, yields

$$
\left[\begin{array}{c}
\dot{\xi}_{1} \\
\dot{\xi}_{2}
\end{array}\right]=\left[\begin{array}{c}
\xi_{2} \\
r\left(m r+M_{H} r+M_{T} r+M_{T} l \cos \left(-\xi_{1}+\theta_{3}^{d}\right)\right) \\
g\left(M_{T} l \sin \left(\theta_{3}^{d}\right)+\left(m r+M_{T} r+M_{H} r\right) \sin \left(\xi_{1}\right)\right)
\end{array}\right] .
$$


In order to establish the relation between (34) and (35), some properties of a double integrator in feedback with a finite-time converging controller are needed.

\section{B. Aside on the Double Integrator}

Consider a scalar double integrator, $\ddot{\eta}(t)=\nu$, and let $\nu:=$ $\psi(\eta, \dot{\eta})$ be any feedback so that Properties P1-P3 of Lemma 1 hold. Let $T_{\text {set }}$ be the settling time function and let $\varphi\left(t, \dot{\eta}_{0}\right)$ denote the solution of the closed-loop system corresponding to the initial condition $\left(0, \dot{\eta}_{0}\right)$. By continuity of the dependence of the solution on the initial conditions, and the fact that $\varphi$ has bounded support, ${ }^{7}$

$$
\begin{aligned}
& \lim _{\epsilon \searrow 0} \sup _{t \geq 0}\left|\varphi\left(t, \epsilon \dot{\eta}_{0}\right)\right|=0 \\
& \lim _{\epsilon \backslash 0} \sup _{t \geq 0}\left|\dot{\varphi}\left(t, \epsilon \dot{\eta}_{0}\right)\right|=0 .
\end{aligned}
$$

Since $\dot{\varphi}$ is a continuous function of $t$, and has bounded support, $\int_{0}^{\infty}\left|\dot{\varphi}\left(t, \dot{\eta}_{0}\right)\right| d t$ exists and is finite. Hence, using (37) and the bounded support property, it follows that

$$
\begin{aligned}
\lim _{\epsilon \backslash 0} & \int_{0}^{\infty}\left|\dot{\varphi}\left(t, \epsilon \dot{\eta}_{0}\right)\right| d t \\
& =\lim _{\epsilon \backslash 0} \int_{0}^{T \operatorname{set}\left(0, \epsilon \dot{m}_{0}\right)}\left|\dot{\varphi}\left(t, \epsilon \dot{\eta}_{0}\right)\right| d t \\
& =\lim _{\epsilon \backslash 0} \int_{0}^{1}\left|\dot{\varphi}\left(t, \epsilon \dot{\eta}_{0}\right)\right| d t=0 .
\end{aligned}
$$

Consider again the scalar double integrator, let $\epsilon>0$, and apply the high gain feedback $\nu=\left(1 / \epsilon^{2}\right) \psi(\eta, \epsilon \dot{\eta})$. Let $\varphi_{\epsilon}\left(t, \dot{\eta}_{0}\right)$ denote the solution for the initial condition $\left(0, \dot{\eta}_{0}\right)$. Then it is straightforward to verify that $\varphi_{\epsilon}\left(t, \dot{\eta}_{0}\right)=\varphi\left(t / \epsilon, \epsilon \dot{\eta}_{0}\right)$, and thus that $\dot{\varphi}_{\epsilon}\left(t, \dot{\eta}_{0}\right)=(1 / \epsilon) \dot{\varphi}\left(t / \epsilon, \epsilon \dot{\eta}_{0}\right)$. Hence, by $(36)$,

$$
\lim _{\epsilon \backslash 0} \sup _{t \geq 0}\left|\varphi_{\epsilon}\left(t, \dot{\eta}_{0}\right)\right|=0
$$

and by (38) and a simple substitution of variables

$$
\lim _{\epsilon \backslash 0} \int_{0}^{\infty}\left|\dot{\varphi}_{\epsilon}\left(t, \dot{\eta}_{0}\right)\right| d t=0
$$

\section{High-Gain Control and the Zero Dynamics}

Once again, let $v_{i}=\psi_{i}\left(y_{i}, \dot{y}_{i}\right), i=1,2$, be any feedbacks for the double integrator so that Properties P1-P3 hold. For any $\epsilon>$ 0 , a simple time scale argument shows that the high gain feedback $v_{i}=\left(1 / \epsilon^{2}\right) \psi_{i}\left(y_{i}, \epsilon \dot{y}_{i}\right)$ still results in Properties P1-P3 being met, and, furthermore, results in the closed-loop settling time function, (25), becoming $T_{\text {set }}^{c l}(y, \dot{y}, \epsilon)=\epsilon T_{\text {set }}^{c l}(y, \epsilon \dot{y})$. With this in mind, apply the feedback

$$
v:=\Psi(\bar{x}):=\left[\begin{array}{l}
\frac{1}{\epsilon^{2}} \psi_{1}\left(\bar{x}_{1}, \epsilon \bar{x}_{3}\right) \\
\frac{1}{\epsilon^{2}} \psi_{2}\left(\bar{x}_{2}, \epsilon \bar{x}_{4}\right)
\end{array}\right]
$$

to (34).

The relationship between the solutions of the closed-loop robot model, (34), and the zero dynamics, (35), is established as

${ }^{7}$ Indeed, the support is $\left[0, T_{\text {set }}\left(0, \epsilon \dot{\eta}_{0}\right)\right]$. follows. Take a point $\bar{x}^{-} \in \hat{S} \cap Z$. Let $\bar{x}^{+}:=\bar{\Delta}\left(\bar{x}^{-}\right)$, where $\bar{\Delta}$ is the representation of $\Delta$ in the coordinates (32). It follows that $\bar{x}_{1}^{+}=0$ and $\bar{x}_{2}^{+}=0$, because (15) is identically zero on $S$. Hence, the analysis of Section V-B is applicable. Letting $\bar{x}\left(t, \bar{x}^{+}\right)$denote the solution of (34) for the initial condition $\bar{x}^{+},(39)$ and (40) imply, respectively,

$$
\lim _{\epsilon \backslash 0} \sup _{t \geq 0}\left|\bar{x}_{i}\left(t, \bar{x}^{+}\right)\right|=0, \quad i=1,2
$$

and

$$
\begin{aligned}
\lim _{\epsilon \backslash 0} & \int_{0}^{\infty}\left|\bar{x}_{j}\left(t, \bar{x}^{+}\right)\right| d t \\
& =\lim _{\epsilon \backslash 0} \int_{0}^{\epsilon T_{\mathrm{sct}}^{c l}\left(0,0, \epsilon \bar{x}_{3}^{+}, \epsilon \bar{x}_{4}^{+}\right)}\left|\bar{x}_{j}\left(t, \bar{x}^{+}\right)\right| d t=0
\end{aligned}
$$

for $j=3,4$. From these two equations, and the fact that $\bar{x}_{3}$ and $\bar{x}_{4}$ appear affinely in $\bar{f}_{5}$, and not at all in $\bar{f}_{6}$, a simple bounding argument $^{8}$ shows that, for $t>0$

$$
\lim _{\epsilon \backslash o} \bar{x}\left(t, \bar{x}^{+}\right)=\left(0,0,0,0, \xi_{1}\left(t, \bar{x}_{5}^{+}, \bar{x}_{6}^{+}\right), \xi_{2}\left(t, \bar{x}_{5}^{+}, \bar{x}_{6}^{+}\right)\right)^{\prime}
$$

where $\xi_{i}\left(t, \bar{x}_{5}^{+}, \bar{x}_{6}^{+}\right), i=1,2$, denotes the solution of the zero dynamics, (35), for the initial condition $\left(\bar{x}_{5}^{+}, \bar{x}_{6}^{+}\right)^{\prime}$.

\section{D. $\lambda$ under High-Gain Control}

It follows that in the high gain limit, that is, as $\epsilon$ tends to zero in (41), the function $\lambda$ from the Poincaré method can be evaluated on the basis of a two-dimensional subsystem, namely, the zero dynamics. Denote the result by $\lambda_{H G}$. This reduction is interesting for several reasons.

1) It brings out the structure of the closed-loop system, and shows that the zero dynamics must encode the notion of a walking cycle.

2) The uniqueness of the solutions of the zero dynamics implies that $\lambda_{H G}$ is strictly monotonic, which partly explains the observed monotonicity in $\lambda$.

3) $\lambda_{H G}$ is as smooth as the data in the problem (for the biped, it is analytic), whereas $\lambda$ is only continuous.

4) The evaluation of $\lambda_{H G}$ is independent of the particular finite-time stabilizing feedback used. Moreover, it can also be computed by replacing $\psi_{i}$ in (41) with a globally exponentially stabilizing feedback, and taking the limit as $\epsilon$ tends to zero; in the limit, the Poincaré return map, $P$, when restricted to $\hat{S} \cap Z$, takes again its values in $S \cap Z$. The consequences of this observation for the study of periodic orbits under nonfinite-time stabilizing feedback control remain to be clarified.

\section{CONCLUSION}

This paper has addressed the problem of establishing the existence of a periodic orbit in a simple biped model, and analyzing its stability properties. The biped model was first formulated as a nonlinear system with impulse effects, evolving in a subset of

${ }^{8}$ Express the solutions in integral form, compute the norm of their difference, and apply the triangle inequality. 
$\mathbb{R}^{6}$. Poincaré's method was then extended to this class of systems. For the biped model considered here, a straightforward application of Poincaré's method would require the computation of a discrete-time map from $\mathbb{R}^{5}$ to $\mathbb{R}^{5}$, which would be difficult to analyze. It was then shown that finite-time converging feedbacks could be used to drive the torso and the swing leg to known functions of the stance leg, and thereby collapse the dimension of the image of the Poincaré map to a one-dimensional set. This leads to an effective analysis tool, which can then be used in design [22]. In the course of the development of these results, it was observed that the zero dynamics of the biped was not invariant under the impact model. It was subsequently shown that its invariance could be recovered under high gain control.

The analysis method developed in the paper is quite general. The next step is to apply it to a more general biped model with knees [42], [21], [18], [19], yielding a seven degree of freedom, under actuated system. It is conjectured that supplementing outputs (15) with hip height and swing foot height objectives will lead to a viable control design with provable stability properties; the horizontal hip position will play the role of $\theta_{1}$ in parameterizing the outputs to be used in the feedback design. It also seems likely that the methods developed here can be applied to other under actuated mechanical systems [40].

The work presented here has assumed a rigid impact model. Non-rigid models have been developed [49] in the context of biped motion. It seems possible that some of the results of the paper can be extended to include such models, though this is more speculative than the previous extension. Finally, many challenging issues exist in running (which has a fly phase) and three dimensional aspects of modeling and control of mechanical biped motion.

\section{APPENDIX A}

\section{MODEL DETAILS}

This appendix completes the equations of the biped model, (1). In the following:

$$
\begin{aligned}
\omega & :=\dot{\theta} \\
s_{1 j} & :=\sin \left(\theta_{1}-\theta_{j}\right), j \in\{2,3\} \\
c_{1 j} & :=\cos \left(\theta_{1}-\theta_{j}\right), j \in\{2,3\} .
\end{aligned}
$$

\section{A. Mechanical Model}

$$
\begin{aligned}
& D=\left[\begin{array}{ccc}
\left(\frac{5}{4} m+M_{H}+M_{T}\right) r^{2} & -\frac{1}{2} m r^{2} c_{12} & M_{T} r l c_{13} \\
-\frac{1}{2} m r^{2} c_{12} & \frac{1}{4} m r^{2} & 0 \\
M_{T} r l c_{13} & 0 & M_{T} l^{2}
\end{array}\right] \\
& C=\left[\begin{array}{ccc}
0 & -\frac{1}{2} m r^{2} s_{12} \omega_{2} & M_{T} r l s_{13} \omega_{3} \\
\frac{1}{2} m r^{2} s_{12} \omega_{1} & 0 & 0 \\
-M_{T} r l s_{13} \omega_{1} & 0 & 0
\end{array}\right] \\
& G=\left[\begin{array}{c}
-\frac{1}{2} g\left(2 M_{H}+3 m+2 M_{T}\right) r \sin \left(\theta_{1}\right) \\
\frac{1}{2} g m r \sin \left(\theta_{2}\right)
\end{array}\right]
\end{aligned}
$$

$$
B=\left[\begin{array}{cc}
-1 & 0 \\
0 & -1 \\
1 & 1
\end{array}\right]
$$

\section{B. Impact Model}

The impact equations (4) and (8), taken together, become

$$
\left[\begin{array}{cc}
D_{e} & -E^{\prime} \\
E & 0
\end{array}\right]\left[\begin{array}{c}
\dot{q}_{e}^{+} \\
F
\end{array}\right]=\left[\begin{array}{c}
D_{e} \dot{q}_{e}^{-} \\
0
\end{array}\right]
$$

where $F=\left(F_{T}, F_{N}\right)^{\prime}$ and the positive-definite symmetric matrix $D_{e}$ has entries

$$
\begin{aligned}
& D_{e}^{11}=\left(\frac{5}{4} m+M_{H}+M_{T}\right) r^{2} \quad D_{e}^{12}=-\frac{1}{2} m r^{2} c_{12} \\
& D_{e}^{13}=M_{T} r l c_{13} \quad D_{e}^{14}=\left(\frac{3}{2} m+M_{H}+M_{T}\right) r \cos \left(\theta_{1}\right) \\
& D_{e}^{15}=-\left(\frac{3}{2} m+M_{H}+M_{T}\right) r \sin \left(\theta_{1}\right) \quad D_{e}^{22}=\frac{1}{4} m r^{2} \\
& D_{e}^{23}=0 \quad D_{e}^{24}=-\frac{1}{2} m r \cos \left(\theta_{2}\right) \quad D_{e}^{25}=\frac{1}{2} m r \sin \left(\theta_{2}\right) \\
& D_{e}^{33}=M_{T} l^{2} \quad D_{e}^{34}=M_{T} l \cos \left(\theta_{3}\right) \\
& D_{e}^{35}=-M_{T} l \sin \left(\theta_{3}\right) \quad D_{e}^{44}=2 m+M_{H}+M_{T} \\
& D_{e}^{45}=0 \quad D_{e}^{55}=2 m+M_{H}+M_{T} .
\end{aligned}
$$

The solvability of (49) is equivalent to the invertibility of the matrix on the left hand side. The invertibility of this matrix follows from the fact that $D_{e}$ is positive definite and $E$ has full rank; indeed, the determinant of the left-hand side of (49) can be computed to be

$$
\begin{array}{r}
\frac{m M_{T} l^{2} r^{4}}{16}\left(3 m+2 M_{T}+4 M_{H}-2 m \cos \left(2 \theta_{1}-2 \theta_{2}\right)\right. \\
\left.-2 M_{T} \cos \left(2 \theta_{2}-2 \theta_{3}\right)\right)
\end{array}
$$

which is nonzero everywhere.

The mapping $\Delta$ is then evaluated by the following steps.

Step 1) Solve (49) for $\dot{q}_{e}^{+}$, and pick-off $\omega^{+}$; since $\dot{q}_{e}^{-}$only depends on $\omega^{-}$(recall that $\dot{z}_{1}^{-}=\dot{z}_{2}^{-}=0$ ), and since the positions do not change during the impact (i.e, $\left.\theta^{+}=\theta^{-}\right)$, the result is $\omega^{+}$expressed as a function of $x^{-}=\left(\theta^{-^{\prime}}, \omega^{-^{\prime}}\right)^{\prime}$.

Step 2) Transform the coordinates so that $\theta_{1}$ corresponds to the stance leg and $\theta_{2}$ to the swing leg; this means swapping the first two position coordinates, and the first two velocity coordinates, respectively.

The final result is

$$
\Delta\left(x^{-}\right):=\left[\begin{array}{llllll}
\theta_{2}^{-} & \theta_{1}^{-} & \theta_{3}^{-} & \omega_{2}^{+}\left(x^{-}\right) & \omega_{1}^{+}\left(x^{-}\right) & \omega_{3}^{+}\left(x^{-}\right)
\end{array}\right]^{\prime} .
$$

The implicit function theorem implies that $\Delta$ is as smooth as the data in (49) and, thus, $\Delta$ is analytic in $x^{-}$.

1) Remarks: a) $\Delta$ is computed in closed form in [22]. b) The no-rebound, no-slip condition of the impact, (8), ensures that the impact results in the end of the swing leg being at rest, and hence, after doing the coordinate transformation, the end of the stance leg will be at rest. c) For the impact model to be valid, it must be verified a posteriori that no-slipping was a valid assumption (that is, $\left|F_{T} / F_{N}\right| \leq \mu$ ), and that the stance leg lifts 
from the ground without interaction (that is, before the coordinate transformation, $\dot{z}_{2}^{+}>0$ ). This was done for all simulations reported in this paper.

\section{Decoupling matrix}

The Lie derivative notation is defined in [30], [37], and [41]

$$
L_{g} L_{f} h=\frac{1}{\operatorname{det}(D)}\left[\begin{array}{ll}
R_{11} & R_{12} \\
R_{21} & R_{22}
\end{array}\right]
$$

where

$$
\begin{aligned}
R_{11}= & \frac{m r^{3}}{4}\left(\frac{5}{4} m r+M_{H} r+M_{T} r-m r\left(c_{12}\right)^{2}+M_{T} l c_{13}\right) \\
R_{12}= & \frac{m r^{3}}{4}\left(\frac{5}{4} m r+M_{H} r+M_{T} r-m r\left(c_{12}\right)^{2}\right. \\
& \left.+2 M_{T} l c_{12} c_{13}\right) \\
R_{21}= & \frac{-m M_{T} l r^{2}}{4}\left(1+2 c_{12}\right)\left(r c_{13}+l\right) \\
R_{22}= & \frac{-M_{T} l r^{2}}{4}\left(5 m l+4 M_{H} l+4 M_{T} l+m r c_{13}\right. \\
& \left.+2 m r c_{12} c_{13}-4 M_{T} l\left(c_{13}\right)^{2}+2 m l c_{12}\right)
\end{aligned}
$$

and

$$
\begin{aligned}
\operatorname{det}(D)= & \frac{m M_{T} r^{4} l^{2}}{4} \\
& \cdot\left(\frac{5}{4} m+M_{H}+M_{T}-m\left(c_{12}\right)^{2}-M_{T}\left(c_{13}\right)^{2}\right) .
\end{aligned}
$$

\section{Zero Dynamics}

In the coordinates used in (19), the zero dynamics is given by

$$
\ddot{\theta}_{1}=\zeta_{0}\left(0,0, \theta_{1}, \dot{\theta}_{1}\right)=: \bar{\zeta}_{a}\left(\theta_{1}\right)+\bar{\zeta}_{b}\left(\theta_{1}\right) \dot{\theta}_{1}^{2}
$$

where

$$
\begin{aligned}
\bar{\zeta}_{a}\left(\theta_{1}\right) & =\frac{g}{r} \frac{\left(2 m+M_{T}+M_{H}\right) r \sin \left(\theta_{1}\right)+M_{T} l \sin \left(\theta_{3}^{d}\right)}{m r+M_{H} r+M_{T} r+M_{T} l \cos \left(\theta_{1}-\theta_{3}^{d}\right)} \\
\bar{\zeta}_{b}\left(\theta_{1}\right) & =\frac{M_{T} l \sin \left(\theta_{1}-\theta_{3}^{d}\right)}{m r+M_{H} r+M_{T^{r}}+M_{T} l \cos \left(\theta_{1}-\theta_{3}^{d}\right)}
\end{aligned}
$$

\section{APPENDIX B}

\section{PROOFS AND TECHNICAL DETAILS}

This appendix collects some of the technical development, in the hope of improving the readability of the main body of the paper.

\section{A. Continuity of $T_{I}$}

Lemma 3: Suppose that $\mathrm{H} 1-\mathrm{H} 3$ hold. Then $T_{I}$ is continuous at points $x_{0}$ where $0<T_{I}\left(x_{0}\right)<\infty$ and $L_{f} H\left(\varphi^{f}\left(T_{I}\left(x_{0}\right), x_{0}\right)\right) \neq 0$.

Proof: Let $\epsilon>0$ be given. Define $\bar{x}:=\varphi^{f}\left(T_{I}\left(x_{0}\right), x_{0}\right)$, and without loss of generality, suppose that $L_{f} H(\bar{x})<0$.
Then, from the definition of $T_{I}$ and $\mathrm{H} 3, H\left(\varphi^{f}\left(t, x_{0}\right)\right)>0$ for all $0 \leq t<T_{I}\left(x_{0}\right)$. This in turn implies that, for any $0<t_{1}<T_{I}\left(x_{0}\right)$

$$
\mu\left(t_{1}\right):=\inf _{0 \leq t \leq t_{1}} \operatorname{dist}\left(\varphi^{f}\left(t, x_{0}\right), S\right)>0
$$

since: a) $\varphi^{f}\left(t, x_{0}\right)$ is continuous in $t$; b) the interval $\left[0, t_{1}\right]$ is compact; and c), by H3, $S$ is closed and equals the zero level set of $H$. By H1, there exists $\bar{\epsilon}>0$ such that $\varphi^{f}$ can be continued on $\left[0, T_{I}\left(x_{0}\right)+\bar{\epsilon}\right]$, [24]. Moreover, since $L_{f} H(\bar{x})<0$, for $\bar{\epsilon}>0$ sufficiently small, $t_{2}:=T_{I}\left(x_{0}\right)+\bar{\epsilon} / 2$ and $x_{2}:=$ $\varphi^{f}\left(t_{2}, x_{0}\right)$, result in $H\left(x_{2}\right)<0$. From $H\left(x_{2}\right)<0$, it follows that $\operatorname{dist}\left(x_{2}, S\right)>0$. If necessary, reduce $\bar{\epsilon}$ so that $0<$ $\bar{\epsilon}<\min \left\{\epsilon, T_{I}\left(x_{0}\right)\right\}$, and define $t_{1}:=T_{I}\left(x_{0}\right)-\bar{\epsilon} / 2$ and $x_{1}:=$ $\varphi^{f}\left(t_{1}, x_{0}\right)$. From (54), $\mu\left(t_{1}\right)>0$. From H2, the solutions depend continuously on the initial conditions. Thus, there exists $\delta>0$, such that, for all $x \in B_{\delta}\left(x_{0}\right), \sup _{0<t<t_{2}} \| \varphi^{f}(t, x)-$ $\varphi^{f}\left(t, x_{0}\right) \|<\min \left\{\operatorname{dist}\left(x_{2}, S\right), \mu\left(t_{1}\right) / 2\right\}$. Therefore, for $x \in$ $B_{\delta}\left(x_{0}\right), t_{1}<T_{I}(x)<t_{2}$, which implies that $\mid T_{I}(x)-$ $T_{I}\left(x_{0}\right) \mid<\epsilon$, establishing the continuity of $T_{I}$ at $x_{0}$.

\section{Distance of a Trajectory to a Periodic Orbit}

Recall that if $\mathcal{O}$ is any periodic orbit that is transversal to $S$, then $\mathcal{O} \subset \tilde{\mathcal{X}}$. For $x \in \tilde{\mathcal{X}}$, define $d(x):=$ $\sup _{0 \leq t \leq T_{I}(x)} \operatorname{dist}\left(\varphi^{-}(t, x), \mathcal{O}\right)$. Note that $d$ vanishes on $\mathcal{O}$. Note also that for $0 \leq t \leq T_{I}(x), \varphi^{-}(t, x)=\varphi^{f}(t, x)$.

Lemma 4: Under $\mathrm{H} 1-\mathrm{H} 3, d: \hat{\mathcal{X}} \rightarrow \mathbb{R}$ is well-defined and is continuous on $\mathcal{O}$.

Proof: For any $x_{0} \in \tilde{\mathcal{X}}, T_{I}\left(x_{0}\right)$ is finite, and $\varphi^{f}\left(t, x_{0}\right)$ is defined on $\left[0, T_{I}\left(x_{0}\right)\right]$. This and the continuity of $\varphi^{f}\left(t, x_{0}\right)$ with respect to t imply that $d\left(x_{0}\right)$ is finite. Next, let $x_{0} \in \mathcal{O}$ and $\epsilon>0$ be given. By definition of $T_{I}, \bar{x}:=\varphi^{f}\left(T_{I}\left(x_{0}\right), x_{0}\right) \in S$. Without loss of generality, suppose that $L_{f} H(\bar{x})<0$. Let $\eta>0$ be such that for all $0<t<\eta, H\left(\varphi^{f}(t, \bar{x})\right)<0$ and $\left\|\bar{x}-\varphi^{f}(t, \bar{x})\right\|<\epsilon / 2$. Such an $\eta$ exists because: (1) H1 implies there exists $\eta>0$ such that $\varphi^{f}$ can be continued on $\left[0, T_{I}\left(x_{0}\right)+\eta\right]$, [24]; (2) $L_{f} H(\bar{x})<0$; and (3) $\varphi^{f}(t, \bar{x})$ depends continuously on $t$. Define $t_{3}:=T_{I}\left(x_{0}\right)+\eta$ and $x_{3}:=\varphi^{f}\left(t_{3}, x_{0}\right)$. By $\mathrm{H} 2$ and Lemma 3, there exists $\delta>0$ such that for all $\tilde{x} \in B_{\delta}\left(x_{0}\right), \sup _{0 \leq t \leq t_{3}}\left\|\varphi^{f}\left(t, x_{0}\right)-\varphi^{f}(t, \tilde{x})\right\|<\epsilon / 2$ and $T_{I}(\tilde{x})<t_{3}$. By the triangle inequality, $\operatorname{dist}\left(\varphi^{f}(t, \tilde{x}), \mathcal{O}\right) \leq$ $\operatorname{dist}\left(\varphi^{f}(t, \tilde{x}), \varphi^{f}\left(t, x_{0}\right)\right)+\operatorname{dist}\left(\varphi^{f}\left(t, x_{0}\right), \mathcal{O}\right)$. Hence, for $\tilde{x} \in B_{\delta}\left(x_{0}\right), \sup _{0 \leq t \leq T_{I}(\tilde{x})} \operatorname{dist}\left(\varphi^{f}(t, \tilde{x}), \mathcal{O}\right) \leq \sup _{0 \leq t \leq t_{3}}$ $\operatorname{dist}\left(\varphi^{f}(t, \tilde{x}), \varphi^{f}\left(t, x_{0}\right)\right)+\sup _{0 \leq t \leq t_{3}} \operatorname{dist}\left(\varphi^{f}\left(t, x_{0}\right), \mathcal{O}\right) \leq$ $\epsilon / 2+\epsilon / 2$, which shows that $\overline{d(\tilde{x})} \leq \epsilon$, and thereby the continuity of $d$ at $x_{0}$.

\section{B. Proof of Theorem 1}

Proof: The first and second statements are immediate. Since the sufficiency portions of the statement c) and d) are straightforward, only necessity is proven here. Suppose that $P\left(x_{0}\right)=x_{0}$, and let $\mathcal{O}$ be the periodic orbit of (11) corresponding to $\Delta\left(x_{0}\right)$. By b), the orbit is transversal to $S$. Let $\epsilon>0$ be given. Since $x_{0}$ is stable in the sense of Lyapunov, for any $\bar{\epsilon}>0$, there exists $\delta(\bar{\epsilon})>0$ such that, for all $k \geq 0$, $\bar{x} \in B_{\delta(\bar{\epsilon})}\left(x_{0}\right) \cap S$, implies $P^{k}(\bar{x}) \in B_{\epsilon}\left(x_{0}\right) \cap S$, where $P^{k}$ is $P$ composed with itself $k$-times. In particular, this implies 
that for all $\bar{x} \in B_{\delta(\bar{\epsilon})}\left(x_{0}\right) \cap S$, there exists a solution $\varphi(t)$ of (11) defined on $[0, \infty)$, such that $\varphi(0)=\Delta(\bar{x})$. Moreover, an upper bound on how far the solution $\varphi$ wanders from the orbit $\mathcal{O}$ is given by

$$
\sup _{t \geq 0} \operatorname{dist}(\varphi(t), \mathcal{O}) \leq \sup _{x \in B_{\bar{c}}\left(x_{0}\right) \cap S} d \circ \Delta(x)
$$

By Lemma 4, since $\mathcal{O}$ is transversal to $S$, and since $\Delta\left(x_{0}\right) \in \mathcal{O}$, $d \circ \Delta$ is continuous at $x_{0}$. Since $d \circ \Delta\left(x_{0}\right)=0$, it follows that there exists $\bar{\epsilon}>0$ such that $\sup _{x \in B_{\bar{c}}\left(x_{0}\right) \cap S} d \circ$ $\Delta(x)<\epsilon$. This bound is valid for all initial conditions in $B_{\delta(\bar{\epsilon})}\left(x_{0}\right) \cap S$. It remains to produce an open neighborhood of $\mathcal{O}$ for which such a bound holds. But this is easily done by taking $\mathcal{V}:=d^{-1}([0, \delta))$, which completes the proof of c). Assume in addition that $\delta(\bar{\epsilon})>0$ was chosen sufficiently small so that $\lim _{k \rightarrow \infty} P^{k}(\bar{x})=x_{0}$. Then by continuity of $d$ and $\Delta$, $\lim _{k \rightarrow \infty} d \circ \Delta\left(P^{k}(\bar{x})\right)=d \circ \Delta\left(x_{0}\right)=0$, from which it easily follows that $\lim _{t \rightarrow \infty} \operatorname{dist}(\varphi(t), \mathcal{O})=0$, proving $\mathrm{d}$ ).

\section{Sufficient Conditions for H1-H2}

The goal is to show that the continuity of the feedback (22) plus Hypotheses $\mathrm{CH} 1-\mathrm{CH} 3$ imply that Hypotheses $\mathrm{H} 1$ and $\mathrm{H} 2$ hold for (24). H1 is immediate. Due to the subgroup property of the flow of a differential equation, it is enough to establish $\mathrm{H} 2$ in a local coordinate chart. Since (2) comes from the second order model, (1), where the matrix $B$ is constant, the input vector fields of (2) commute and the dimension of their span is constant. These two facts plus the invertibility of the decoupling matrix (Hypothesis $\mathrm{CH} 1$ ) imply that, about any point $x_{0} \in \mathcal{X}$, the system (24) can be locally transformed into [30], [37], [41]

$$
\begin{aligned}
\dot{\zeta}_{1} & =\zeta_{2} \\
\dot{\zeta}_{2} & =\Psi\left(\zeta_{1}, \zeta_{2}\right) \\
\dot{z} & =\Gamma\left(\zeta_{1}, \zeta_{2}, z\right)
\end{aligned}
$$

where $\zeta_{1}:=y, \zeta_{2}:=\dot{y}, \Psi$ is given by (22) and $\Gamma$ is an analytic function of its arguments (the analyticity comes from that of (1)). In particular, $\Gamma$ is locally Lipschitz continuous.

Thus, in these coordinates, the system is expressed as a cascade of a system that satisfies $\mathrm{H} 2$ feeding forward into a system that is locally Lipschitz. The Gronwal inequality [34] can therefore be used to establish that $\mathrm{H} 2$ holds for the cascade. This is summarized in the lemma below.

Lemma 5: For the closed-loop system (24), Hypotheses $\mathrm{CH} 1-\mathrm{CH} 3$ and the continuity of (22) imply Hypotheses H1 and $\mathrm{H} 2$.

\section{ACKNOWLEDGMENT}

The first author wishes to thank D. Koditschek for stimulating conversations on the control of under actuated mechanical systems. This work was initiated while the first author was on sabbatical at GRAViR-LSIIT; he thanks E. Ostertag for his kind hospitality.

\section{REFERENCES}

[1] R. McN. Alexander, "Three uses for springs in legged locomotion," Int. J. Robot. Res, vol. 9, no. 2, pp. 53-61, 1990.
[2] V. I. Babitsky, Theory of Vibro-Impact Systems and Applications. Berlin, Germany: Springer-Verlag, 1998.

[3] D. D. Bainov and P. S. Simeonov, Systems with Impulse Effects: Stability, Theory and Applications. Chichester, U. K.: Ellis-Horwood, 1989.

[4] S. P. Bhat and D. S. Bernstein, "Continuous finite-time stabilization of the translational and rotational double integrators," IEEE Trans. Automat. Contr., vol. 43, pp. 678-682, May 1998.

[5] S. P. Bhat and D. S. Bernstein, "Finite-time stability of continuous autonomous systems,", 1998.

[6] S. P. Bhat and D. S. Bernstein, "Finite-time stability of homogeneous systems,", 1998.

[7] R. M. Brach, "Rigid body collisions," J. Appl. Mech., vol. 56, pp. 133-138, 1989.

[8] B. Brogliato, Nonsmooth Impact Dynamics: Models, Dynamics and Control. London, U. K.: Springer-Verlag, 1996.

[9] M. Bühler, D. E. Koditschek, and P. J. Kindlmann, "Planning and control of a juggling robot," Int. J. Robot. Res., vol. 13, no. 2, pp. 101-118, 1994.

[10] G. Cabodevilla, G. Abba, and H. Sage, "Energetically near optimal gait for a biped robot with double supporting phases," in Proc. Third France-Japan Congr. First Europe-Asia Congr. Mechatronics, Besancon, France, Oct. 1996, pp. 958-961.

[11] G. Cabodevilla, N. Chaillet, and G. Abba, "Energy-minimized gait for a biped robot," in Autonome Mobile Systeme. Berlin, Germany: Springer-Verlag, 1995, pp. 90-99.

[12] C. Canudas, L. Roussel, and A. Goswani, "Periodic stabilization of a 1-dof hopping robot on nonlinear compliant surface," in Proc. IFAC Symp. Robot Control, Nantes, France, Sept. 1997, pp. 405-410.

[13] N. Chaillet, G. Abba, and E. Ostertag, "Double dynamic modeling and computed-torque control of a biped robot," in Proc. IEEE/RSJ Int. Conf. Intelligent Robotics Systems, Munich, Germany, Sept. 1994, pp. 1149-1153.

[14] C. Chevallereau, A. Formal'sky, and B. Perrin, "Control of a walking robot with feet following a reference trajectory derived from ballistic motion," in Proc. IEEE Int. Conf. Robotics Automation, Albuquerque, NM, Apr. 1997, pp. 1094-1099.

[15] B. Espiau and A. Goswani, "Compass gait revisited," in Proc. IFAC Symp. Robot Control, Capri, Italy, Sept. 1994, pp. 839-846.

[16] C. Francois and C. Samson, "A new approach to the control of the planar one-legged hopper," Int. J. Robot. Res., vol. 17, no. 11, pp. 1150-1166, 1998.

[17] Y. Fujimoto and A. Kawamura, "Simulation of an autonomous biped walking robot including environmental force interaction," IEEE Robot. Automat. Mag., pp. 33-42, June 1998.

[18] J. Furusho and M. Masubuchi, "Control of a dynamical biped locomotion system for steady walking," J. Dyn. Syst., Meas., Control, vol. 108, pp. 111-118, 1986.

[19] J. Furusho and A. Sano, "Sensor-based control of a nine-link biped," Int. J. Robot. Res., vol. 9, no. 2, pp. 83-98, 1990.

[20] A. Goswani, B. Espiau, and A. Keramane, "Limit cycles and their stability in a passive bipedal gait," in Proc. IEEE Int. Conf. Robotics Automation, Minneapolis, MN, Apr. 1996, pp. 246-251.

[21] A. A. Grishin, A. M. Formal'sky, A. V. Lensky, and S. V. Zhitomirsky, "Dynamical walking of a vehicle with two telescopic legs controlled by two drives," Int. J. Robot. Res., vol. 13, no. 2, pp. 137-147, 1994.

[22] J. W. Grizzle, G. Abba, and F. Plestan, "Proving asymptotic stability of a walking cycle for a five dof biped robot model," in Proc. 2 nd Int. Conf. Climbing and Walking Robots, Portsmouth, U. K., Sept. 1999, pp. 69-81.

[23] V. T. Haimo, "Finite time controllers," SIAM J. Contr. Optim., vol. 24, no. 4, pp. 760-770, 1986.

[24] P. Hartman, Ordinary Differential Equations, 2nd ed. Boston, MA: Birkhauser, 1982

[25] H. Hemami and B. F. Wyman, "Modeling and control of constrained dynamic systems with application to biped locomotion in the frontal plan," IEEE Trans. Automat. Contr., vol. 24, pp. 526-535, Mar. 1979.

[26] Y. Hurmuzlu, "Dynamics of bipedal gait—Part 1: Objective functions and the contact event of a planar five-link biped," J. Appl. Mech., vol. 60, pp. 331-336, June 1993.

[27] —, "Dynamics of bipedal gait—Part 2: Stability analysis of a planar five-link biped," J. Appl. Mech., vol. 60, pp. 337-343, June 1993.

[28] Y. Hurmuzlu and D. B. Marghitu, "Rigid body collisions of planar kinematic chains with multiple contact points," Int. J. Robot. Res., vol. 13, no. 1, pp. 82-92, 1994.

[29] Y. Hurmuzlu and D. Moskowitz, "The role of impact in the stability of bipedal locomotion," Dyn. Stabil. Syst., vol. 1, no. 3, pp. 217-234, 1986.

[30] A. Isidori, Nonlinear Control Systems: An Introduction, 2nd ed. Berlin, Germany: Springer-Verlag, 1989. 
[31] S. Kajita and K. Tani, "Experimental study of biped dynamic walking in the linear inverted pendulum mode," in Proc. IEEE Int. Conf. Robotics Automation, Nagoya, Japan, May 1995, pp. 2885-2891.

[32] S. Kajita, T. Yamaura, and A. Kobayashi, "Dynamic walking control of biped robot along a potential energy conserving orbit," IEEE Trans. Robot. Automat., vol. 8, pp. 431-437, Aug. 1992.

[33] R. Katoh and M. Mori, "Control method of biped locomotion giving asymptotic stability of trajectory," Automatica, vol. 20, no. 4, pp. 405-414, 1984

[34] H. K. Khalil, Nonlinear Systems-2nd Edition. Englewood Cliffs, NJ: Prentice-Hall, 1996.

[35] D. D. Koditschek and M. Buhler, "Analysis of a simplified hopping robot," Int. J. Robot. Res., vol. 10, no. 6, pp. 587-605, 1991.

[36] N. Manamani, N. N. Gauthier, and N. K. M'Sirdi, "Sliding mode control for pneumatic robot leg," in Proc. European Control Conf., Brussels, Belgium, July 1997.

[37] T. Marino and P. Tomei, Nonlinear Control Design. London, U. K.: Prentice-Hall, 1995.

[38] T. McGeer, "Passive dynamic walking," Int. J. Robot. Res., vol. 9, no. 2, pp. 62-82, 1990.

[39] K. Mitobe, N. Mori, K. Aida, and Y. Nasu, "Nonlinear feedback control of a biped walking robot," in Proc. IEEE Int. Conf. Robotics Automation, Nagoya, Japan, May 1995, pp. 2865-2870.

[40] J. Nakanishi, T. Fukuda, and D. E. Koditschek, "Preliminary studies of a second generation brachiation robot controller," in Proc. IEEE Int. Conf. Robotics Automation, Albuquerque, NM, Apr. 1997, pp. 2050-2056.

[41] H. Nijmeijer and A. J. van der Schaft, Nonlinear Dynamical Control Systems. Berlin, Germany: Springer-Verlag, 1989.

[42] J. H. Park and K. D. Kim, "Biped robot walking using gravity-compensated inverted pendulum mode and computed torque control," in Proc. IEEE Int. Conf. Robotics Automation, Leuven, Belgium, May 1998, pp. 3528-3533.

[43] T. S. Parker and L. O. Chua, Practical Numerical Algorithms for Chaotic Systems. New York: Springer-Verlag, 1989.

[44] F. Pfeiffer and C. Glocker, Multi-Body Dynamics with Unilateral Constraints. New York: Wiley, 1996.

[45] J. Pratt and G. Pratt, "Intuitive control of a planar bipedal walking robot," in Proc. IEEE Int. Conf. Robotics Automation, Leuven, Belgium, May 1998, pp. 2014-2021.

[46] M. H. Raibert, "Legged robots," Сотmun. АCM, vol. 29, no. 6, pp. 499-514, 1986

[47] M. H. Raibert, S. Tzafestas, and C. Tzafestas, "Comparative simulation study of three control techniques applied to a biped robot," in Proc. IEEE Int. Conf. Systems, Man, Cybernetics-System Engineering Service Humans, Le Touquet, France, Oct. 1993, pp. 494-502.

[48] M. Rostami and G. Bessonnet, "Impactless sagittal gait of a biped robot during the single support phase," in Proc. IEEE Int. Conf. Robotics Automation, Leuven, Belgium, May 1998, pp. 1385-1391.

[49] L. Roussel, "Génération de trajectoires de marche optimales pour un robot bipède," $\mathrm{Ph}$.D. dissertation, Institut National Polytechnique, Grenoble, France, Nov. 1998.

[50] A. Sano and J. Furusho, "Realization of natural dynamic walking using the angular momentum information," in Proc. IEEE Int. Conf. Robotics Automation, Cincinnati, OH, May 1990, pp. 1476-1481.

[51] U. Saranli, W. J. Schwind, and D. E. Koditschek, "Toward the control of a multi-jointed, monoped runner," in Proc. IEEE Int. Conf. Robotics and Automation, Leuven, Belgium, May 1998, pp. 2676-2682.

[52] C. L. Shih and W. A. Gruver, "Control of a biped robot in the doublesupport phase," IEEE Trans. Syst., Man, Cybern., vol. 22, pp. 729-735, 1992.

[53] A. C. Smith and M. D. Berkemeier, "The motion of a finite-width wheel in 3d," in Proc. IEEE Int. Conf. Robotics Automation, Leuven, Belgium, May 1998, pp. 2345-2350.
[54] M. W. Spong and M. Vidyasagar, Robot Dynamics and Control. New York: Wiley, 1989.

[55] D. E. Stewart, "Convergence of a time-stepping scheme for rigid body dynamics and resolution of painlevé's problem," Arch. Rational Mech. Anal., vol. 145, pp. 215-260, 1998.

[56] B. Thuilot, A. Goswani, and B. Espiau, "Bifurcation and chaos in a simple passive bipedal gait," in Proc. IEEE Int. Conf. Robotics Automation, Albuquerque, NM, Apr. 1997, pp. 792-798.

[57] R. Q. van der Linde, "Active leg compliance for passive walking," in Proc. IEEE Int. Conf. Robotics Automation, Leuven, Belgium, May 1998, pp. 2339-2344.

[58] M. Vukobratovic, B. Borovac, D. Surla, and D. Stokic, Biped Locomotion. Berlin, Germany: Springer-Verlag, 1990

[59] H. Ye, A. N. Michel, and L. Hou, "Stability theory for hybrid dynamical systems," IEEE Trans. Automat. Contr., vol. 43, pp. 461-474, Apr. 1998.

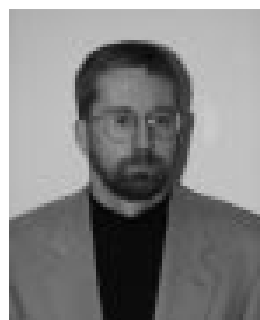

Jessy W. Grizzle received the Ph.D. degree in electrical engineering from the University of Texas, Austin, in 1983.

Since September 1987, he has been with the University of Michigan, Ann Arbor, where he is currently a Professor of Electrical Engineering and Computer Science. He has been an Associate Editor of Systems and Control Letters and has worked on a wide range of theoretical issues in nonlinear control and observer design. He is actively pursuing applications of system theory in the automotive industry and semiconductor

manufacturing

Prof. Grizzle has been an Associate Editor of the IEEE Transactions on Automatic Control and, from 1997 to 1999, served on the Board of Governors of the IEEE Control Systems Society.

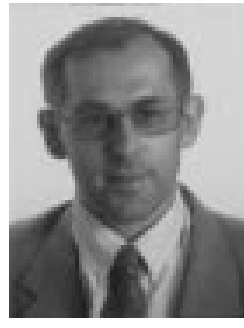

Gabriel Abba entered the Ecole Normale Supérieure de Cachan, France in 1979, and received agrégation from the Ministry of Education in Electrical Engineering in 1982. He received the Ph.D. degree in electronics and robotics from the University of Paris XI-Orsay, Paris, France in1986.

$\mathrm{He}$ is with LGIPM, Universit de Metz, Yutz, France. His research interests include development, modeling and control of robots, especially control of legged robots, visual servoing, and control of high-speed drives for industrial applications.

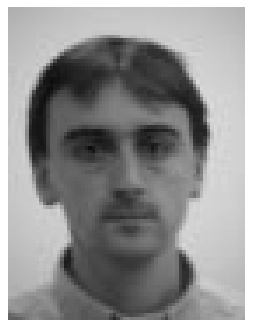

Franck Plestan received the Ph.D. degree in Automatic Control from the Ecole Centrale de Nantes, Nates, France, in 1995.

From September 1996 to August 2000, he was with the Université Louis Pasteur, Strasbourg, France. In September 2000, he joined IRCCyN, Ecole Centrale de Nantes, France. His research interests include nonlinear control, theoretical aspects of nonlinear observer design and, more recently, control of legged robots. 